\title{
Evaluation of Probiotics for Warfighter Health and Performance
}

\author{
Richard T. Agans 1,2, Grace E. Giles ${ }^{3}$, Michael S. Goodson ${ }^{4}$, J. Philip Karl'5, \\ Samantha Leyh ${ }^{4,6}$, Karen L. Mumy ${ }^{2}$, Kenneth Racicot ${ }^{3}$, and Jason W. Soares ${ }^{3 *}$ on behalf \\ of the Tri-Service Microbiome Consortium
}

${ }^{1}$ Henry M. Jackson Foundation for the Advancement of Military Medicine, Bethesda, MD, United States, ${ }^{2}$ Naval Medical Research Unit Dayton, Environmental Health Effects Laboratory, Dayton, OH, United States, ${ }^{3}$ Soldier Performance Optimization Directorate, U.S. Army Combat Capabilities Development Command - Soldier Center, Natick, MA, United States, ${ }^{4}$ Air Force Research Laboratory, 711th Human Performance Wing, Wright Patterson Air Force Base, Dayton, $\mathrm{OH}$, United States, ${ }^{5}$ Military Nutrition Division, U.S. Army Research Institute of Environmental Medicine, Natick, MA, United States, ${ }^{6}$ Oak Ridge Institute for Science and Education, Wright Patterson Air Force Base, Oak Ridge, TN, United States

The probiotic industry continues to grow in both usage and the diversity of products available. Scientific evidence supports clinical use of some probiotic strains for certain gastrointestinal indications. Although much less is known about the impact of probiotics in healthy populations, there is increasing consumer and scientific interest in using probiotics to promote physical and psychological health and performance. Military men and women are a unique healthy population that must maintain physical and
OPEN ACCESS

Edited by:

Jennie Cecile Brand-Miller, University of Sydney, Australia

Reviewed by:

Mary Ellen Sanders, Dairy \& Food Culture Technologies, United States

Emilia Vassilopoulou, International Hellenic

University, Greece

*Correspondence:

Jason W. Soares Jason.w.soares.civ@mail.mil

Specialty section: Nutrition and Metabolism

a section of the journal

Frontiers in Nutrition

Received: 13 March 2020 Accepted: 24 April 2020

Published: 09 June 2020

Citation:

Agans RT, Giles GE, Goodson MS, Karl JP, Leyh S, Mumy KL, Racicot K and Soares JW (2020) Evaluation of Probiotics for Warfighter Health and

Performance. Front. Nutr. 7:70. doi: 10.3389/fnut.2020.00070 This article was submitted to psychological health in order to ensure mission success. In this narrative review, we examine the evidence regarding probiotics and candidate probiotics for physical and/or cognitive benefits in healthy adults within the context of potential applications for military personnel. The reviewed evidence suggests potential for certain strains to induce biophysiological changes that may offer physical and/or cognitive health and performance benefits in military populations. However, many knowledge gaps exist, effects on health and performance are generally not widespread among the strains examined, and beneficial findings are generally limited to single studies with small sample sizes. Multiple studies with the same strains and using similar endpoints are needed before definitive recommendations for use can be made. We conclude that, at present, there is not compelling scientific evidence to support the use of any particular probiotic(s) to promote physical or psychological performance in healthy military personnel. However, plausibility for physical and psychological health and performance benefits remains, and additional research is warranted. In particular, research in military cohorts would aid in assessing the value of probiotics for supporting physical and psychological health and performance under the unique demands required of these populations.

Keywords: microbiota, probiotics, performance, cognition, warfighter, microbiome, physical, nutrition

\section{INTRODUCTION}

Health, readiness, and performance (defined as the ability to meet mission demands) are important measures within the military. The men and women who serve are held to stringent standards within each of those metrics throughout their military careers, ensuring that forces retain high capability (1-3). Military personnel are also often required to operate under conditions of sub-optimal 
sleep and/or nutrition, in extreme environments, and under elevated stress. In these situations, failure to perform optimally could mean the difference between mission success and failure. Some programs exist to promote healthy lifestyles, such as the Army's Performance Triad program. This program focuses on getting optimal sleep, activity, and nutrition in order to achieve the health and readiness goals required to ensure mission success. Nevertheless, the desire to optimize individual performance has been reported as a driving factor for service members to take dietary supplements, and significantly more military personnel are now using dietary supplements than the general population (69\% compared to $50 \%$, respectively) (4-7).

Live microorganisms are increasingly being included in dietary supplements resulting in a global industry currently valued at over $\$ 40$ billion and forecasted to amass $\$ 64$ billion in sales by 2023 (8). Although foods containing bacteria and/or their metabolites have long been recognized for their "health preserving" properties, interest in isolating and consuming certain bacteria began for researchers in the late twentieth century (9-11). At that time, the term "probiotic" was created. The definition of probiotic has evolved over time, with recent consensus settling on "live microorganisms that, when administered in adequate amounts, confer a health benefit on the host" (12). Inherent in this definition is that not all microorganisms can be considered as probiotic, and correct use of the term requires strain level identification, empirical evidence of health benefits in the target host, and the delivery of live microbes in adequate doses to elicit the health benefit.

The most common focus for probiotic research and development has been microbes that are administered orally to be delivered to the gastrointestinal (GI) tract. These ingested microbes compete and interact with the bacteria, archaea, viruses, and eukaryotes which constitute the commensal microbial content of the GI tract known as the "gut microbiome." This research has led to the development of probiotics that have demonstrated efficacy in some populations suffering from upper respiratory tract infections (URTI), and GI-related maladies including travelers and acquired acute diarrhea, irritable bowel syndrome (IBS), inflammatory bowel disease (e.g., IBD, Crohn's disease, etc.), and lactose intolerance (10, 13-15). The putative mechanisms underlying health benefits of probiotics are not fully resolved, but are thought to include those noted in Figure 1. Importantly, some of those mechanisms and resulting health benefits are strain-specific whereas others may be more




widespread across probiotic strains (12). Therefore, it cannot be assumed that all probiotics will have the same effects. Products containing live microorganisms are also being developed and sold to support the health of extra-intestinal organs such as the vaginal-tract, lung, and skin. As a result, avast array of products containing live microorganisms including juices, diet bars, infant formulas, waters, chewing gum, sweeteners, pizza, toothpaste, and cosmetics are now available to consumers (16). These products are marketed toward individuals seeking to improve their mood, skincare, gut and vaginal health, and a myriad of other aspects of physical and psychological health and wellness, and many of these products claim to contain probiotics (17-19).

Dietary supplements, foods, and other probiotic-containing products are not regulated in the same manner as drugs, which require evidence of clinical efficacy for curing, treating, preventing or mitigating a disease, and do not require premarket review by the Federal Drug Administration (FDA). Rather, dietary supplements are permitted to make general "well-being" claims that do not require FDA approval. If a product labeled as a dietary supplement makes a claim involving the cure, treatment, prevention or mitigation of disease, that product is considered to be an unapproved drug and is subject to FDA action (20). The extent to which claims on commercial probiotic products are substantiated is not clear, but recent retail surveys found that only about $35 \%$ of probiotic supplements and $50 \%$ of probiotic foods could be clearly linked to any health benefit $(21,22)$.

Previous reviews have examined probiotic use in at-risk or health-compromised individuals, discussed regulatory questions, and attempted to refine the definition of probiotic products $(16,18,19,23,24)$, but few have considered the potential benefit (or harm) of probiotic use in military personnel specifically (25). Given the growing presence of these products in the marketplace, the high use of dietary supplements by military personnel, and the potential for (and marketing of) probiotic products to benefit general health, there is likely to be increased interest by military personnel in using probiotics.

This review was conducted to assess the current body of evidence regarding the impact of probiotics in healthy adults on outcomes directly relevant to health and performance of military personnel, and to identify knowledge gaps where further research is needed to establish probiotic efficacy in this population. Given the broad scope and unique population, this review is intended to present a narrative overview of the evidence base with respect to military relevance. The review is organized using two health and performance core areas of importance to the military: physical and psychological domains, with specific sub-elements discussed for each domain, Figure 2.

\section{SEARCH CRITERIA}

Systematic search criteria were not used for this narrative review. However, to provide a comprehensive evaluation of the evidence base, authors conducted separate literature searches for each topic area included in the review using PubMed and/or Google Scholar. Searches used the logical operator "OR" between probiotic-related terms (e.g., "probiotic,"

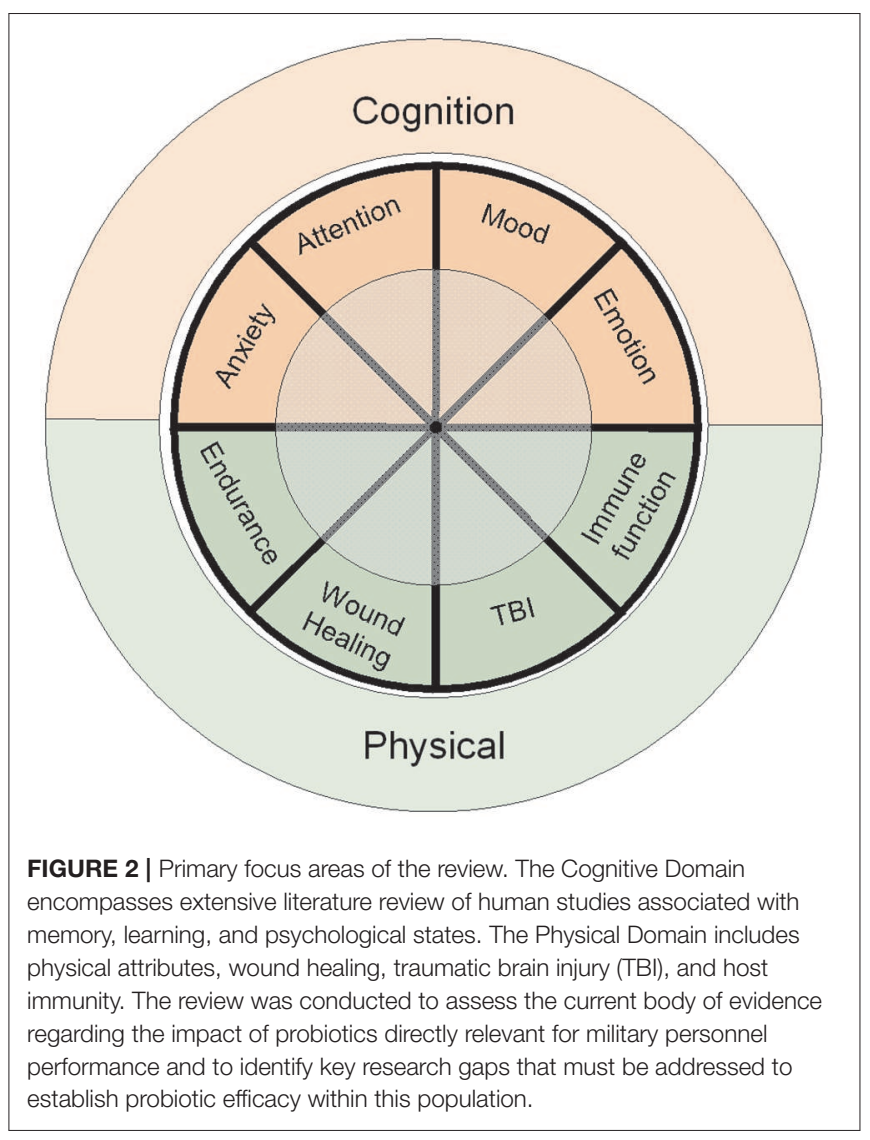

"Lactobacillus," "Bifidobacterium," "gut microbiome") and the logical operator "AND" between the probiotic-related terms and topic-specific terms. For example, for the cognition topic area, either the cognition search modifier cogniti* (i.e., "cognition," "cognitive"), affective (i.e., "mood," "emotion," "anxiety," "depression," "stress"), or cognitive tasks (e.g., "stroop task") were used. Reference lists of relevant narrative and systematic reviews were also manually searched. All searches were completed prior to September 2019; however, relevant studies published after that date were included if the authors were aware of their publication. Human intervention trials published in peer-reviewed literature were considered for inclusion irrespective of study design. Studies published solely in abstract form or in gray literature were not considered.

\section{PHYSICAL DOMAIN}

Exercise, especially of high-intensity or sustained for long periods of time, increases physiological stress, and metabolic demands (26). Those effects can induce transient oxidative stress, changes in intestinal permeability, and systemic inflammation (27). When recovery is insufficient, immune function can also be compromised. Probiotics have been proposed as a strategy for mitigating these effects through reduction of reactive oxygen/nitrogen species and inflammation, and for 
promoting intestinal barrier integrity and immune function (2831). As such, multiple studies have examined whether probiotics and candidate probiotics can promote exercise performance, post-exercise recovery, and immune function during exercise training (32).

\section{Exercise Performance}

Several studies have examined effects of various probiotics and candidate probiotics on exercise performance, and endurance performance in particular (Table 1). These studies have used both multi- and single-strain formulations, included tests of endurance, strength and power, and been conducted in a variety of populations including both endurance and skill athletes (33, 38, 40-42), and sedentary adults (37). Several have reported favorable effects. For example, increases in time to exhaustion have been reported with both multi-stain and single-strain products $(35,39)$, and, in one study, Lactobacillus plantarum PS128 supplementation reduced oxidative stress and improved performance during a triathlon (36). Confirmatory studies; however, are rare, and more often, studies have failed to demonstrate beneficial effects of probiotics on exercise performance. Indeed, a recent position stand on the use of probiotics in athletes concluded that while studies using multistrain products seem to more often demonstrate benefit for aerobic performance than single-strain products, the majority of studies have found no effect on aerobic performance (32).

\section{Exercise-Induced Muscle Damage and Recovery}

Exhaustive and/or unaccustomed exercise induces temporary muscle damage resulting in delayed onset muscle soreness, loss of muscle strength and power, decreased muscle function, and impaired physical performance which require a complex cascade of mechanisms for repair and recovery (43). The combined effects of stress, inadequate rest and recovery, and suboptimal nutrition in military populations during and between exercise bouts can compromise or prolong recovery from exercise-induced muscle damage resulting in performance decrements and injury (44). Emerging evidence supports the possibility of a "gut microbiome-gut-muscle axis," by which gut microbes influence muscle damage, growth, and repair through multiple interrelated mechanisms. These mechanisms are thought to include modulation of nutrient absorption, intestinal permeability, anabolic hormones (e.g., insulin-like growth factor-1), inflammation, immune function, and myocellular signaling (45-47). Accordingly, recent studies have begun to explore the efficacy of probiotics for reducing muscle damage and accelerating muscle repair and recovery (Table 2). Results of those studies have been largely inconclusive, but suggest some promise for specific probiotic strains. For example, Jager et al. reported that supplementation with Bacillus coagulans GBI-30, 6086 (BC30) and the protein casein (relative to casein alone) reduced soreness, attenuated increases in markers of muscle damage, and prevented a $5 \%$ decrease in some, but not all, measures of physical performance following a single bout of muscle damaging exercise in recreationallyactive young men (49). However, the non-randomized, prepost study design precluded determining whether the effects were due to BC30 supplementation or were simply a training effect. In a study of elite soldiers, BC30 in combination with $\beta$-hydroxy- $\beta$-methylbutyrate, prevented a $39 \%$ decrease in one of four measures of muscle integrity, but did not impact circulating markers of muscle damage or inflammation during a $40-\mathrm{d}$ military training exercise compared to $\beta$ hydroxy- $\beta$-methylbutyrate alone $(48,51)$. In another study, daily supplementation with the combination of Streptococcus thermophilus FP4 and Bifidobacterium breve BR03 relative to placebo improved physical performance by $\sim 10 \%$ without impacting perceived soreness, circulating markers of muscle damage, or muscle swelling following a muscle-damaging exercise bout in resistance-trained young men (50).

\section{Training Stress and Respiratory Immunity}

Several groups have published studies examining evidence concerning effects of probiotics on respiratory immune function in athletes. Recently, systematic reviews have been conducted on the evidence presented in these studies. King et al. reviewed 21 clinical trials involving 4,273 participants consuming Lactobacillus or Bifidobacterium probiotics (52). Similarly, Hao et al. performed a systematic review on 13 clinical trials, performing meta-analysis on 12 (3,270 participants) ranging from children to older adults, taking Bifidobacterium, Lactobacillus, and Streptococcus probiotics (13). Both groups concluded that the evidence presented supports probiotic benefit for reducing numbers of URTI episodes, duration, and related work absences $(13,52)$. However, Hao et al. raised concern over quality of evidence in their review, citing "low and very low quality of evidence" (13). Of particular relevance to this paper are those studies that focused on the severity and incidence of URTIs in healthy adults and athletes.

Intense training and exercise, especially when recovery is insufficient, can increase risk of immune impairment and URTIs (53-55). Following recurring periods of physical activity, which can be common in some military personnel, is a period termed the "open window" of immune suppression when it is suggested that pathogens are more likely to invade and establish infection (53). In fact, respiratory infectious diseases account for up to $30 \%$ of infection related military hospitalizations, and have been estimated to impact up to 80,000 recruits and 600,000 active duty service members each year (56). Ultimately, this has resulted in up to 27,000 lost training days and 95,241 lost duty days annually (56). Lost duty and training time can drastically reduce military strength and readiness.

Several studies show improvements in biomarkers or biochemistries associated with immune function following probiotic intervention. However, the overall evidence is mixed (Table 3). Seven studies have reported some benefit of probiotics on URTI symptoms, severity, or duration in athletes. Dose, probiotic strain, single- vs. multi-strain formulations, and duration (2-23 week) vary greatly across those studies. All studies showing some respiratory improvement included organisms from the genus Lactobacillus. What is lacking are associations 
TABLE 1 | Probiotic influence on physical performance in healthy individuals.

\begin{tabular}{|c|c|c|c|c|c|}
\hline References & Pop. $^{a}$ & Study design & Probiotic administration & Duration & Performance measures and results \\
\hline Carbuhn et al. (33) & $\begin{array}{l}16 \text { collegiate } \\
\text { swimmers; age } \\
\text { not reported }\end{array}$ & $\mathrm{DB}, \mathrm{RCT}$ & $\begin{array}{l}\text { B. longum } 356241 \times 10^{9} \mathrm{CFU} / \mathrm{d} \text { vs. } \\
\text { placebo during intensified } \\
\text { training period }\end{array}$ & 6 weeks & $\begin{array}{l}\text { Aerobic performance: no differences } \\
\text { Anaerobic performance: no differences } \\
\text { Lower body power: no differences } \\
\text { Inflammation: no differences } \\
\text { Immunity: no differences } \\
\text { Cognitive stress: improved w/ probiotic }\end{array}$ \\
\hline Huang et al. (35) & $\begin{array}{l}16 \text { amateur runners } \\
20-40 \text { years }\end{array}$ & $\mathrm{DB}, \mathrm{RCT}$ & $\begin{array}{l}\text { L. plantarum TWK10. } 1 \times 10^{11} \\
\text { CFU/d vs. placebo }\end{array}$ & 6 weeks & $\begin{array}{l}\text { Aerobic performance: Increased run time to } \\
\text { exhaustion } \\
\text { Muscle damage (Creatine Kinase): no differences }\end{array}$ \\
\hline $\begin{array}{l}\text { Huang et al. (36) } \\
\text { (study 1) }\end{array}$ & $\begin{array}{l}18 \text { triathletes } \\
19-24 \text { years }\end{array}$ & $\mathrm{DB}, \mathrm{RCT}$ & $\begin{array}{l}\text { L. plantarum PS128 } 3 \times 10^{10} \mathrm{CFU} / \mathrm{d} \\
\text { vs. placebo during triathlon training }\end{array}$ & 4 weeks & $\begin{array}{l}\text { Inflammation: improved w/probiotic } \\
\text { Muscle damage: no differences }\end{array}$ \\
\hline Ibrahim et al. (37) & $\begin{array}{l}21 \text { sedentary young } \\
\text { men; } 21 \pm 2 \text { years }\end{array}$ & $\begin{array}{l}\mathrm{DB}, \mathrm{RCT} \\
\text { parallel }\end{array}$ & $\begin{array}{l}\text { L. acidophilus BCMC 12130, L. } \\
\text { casei BCMC 12313, L. lactis BCMC } \\
12451, \text { B. bifidum BCMC 02290, B. } \\
\text { infantis BCMC } 02129, \text { B. longum } \\
\text { BCMC } 02120\left(6 \times 10^{10} \text { CFU/d) vs. }\right. \\
\text { placebo during circuit } \\
\text { training program }\end{array}$ & 12 weeks & $\begin{array}{l}\text { Muscle strength and power: no differences } \\
\text { Serum inflammation markers: no differences }\end{array}$ \\
\hline Marshall et al. (38) & $\begin{array}{l}32 \text { endurance } \\
\text { runners } \\
\text { 23-53 years }\end{array}$ & & $\begin{array}{l}\text { Multi-strain probiotic }{ }^{\mathrm{b}} \text { vs. } \\
\text { multi-strain probiotic }+ \text { glutamine }^{\mathrm{c}} \\
\text { vs. placebo during ultramarathon } \\
\text { training }\end{array}$ & 12weeks & $\begin{array}{l}\text { Aerobic fitness: no differences } \\
\text { Aerobic performance: no differences }\end{array}$ \\
\hline Toohey et al. (40) & $\begin{array}{l}23 \text { collegiate } \\
\text { athletes } \\
20 \pm 1 \text { years }\end{array}$ & $\mathrm{DB}, \mathrm{RCT}$ & $\begin{array}{l}\text { B. subtilis DE111 } 5 \times 10^{9} \mathrm{CFU} / \mathrm{d} \text { vs. } \\
\text { placebo during resistance training } \\
\text { program }\end{array}$ & 10 weeks & $\begin{array}{l}\text { Lower and upper body strength: no differences } \\
\text { Lower body power: no differences } \\
\text { Agility: no differences } \\
\text { Body composition: improved w/probiotic }\end{array}$ \\
\hline Townsend et al. (41) & $\begin{array}{l}25 \text { collegiate } \\
\text { baseball players } \\
20 \pm 1 \text { years }\end{array}$ & $\mathrm{DB}, \mathrm{RCT}$ & $\begin{array}{l}\text { B. subtilis DE111 } 1 \times 10^{9} \mathrm{CFU} / \mathrm{d} \text { vs. } \\
\text { placebo during offseason training }\end{array}$ & 12 weeks & $\begin{array}{l}\text { Lower body strength: no differences } \\
\text { Lower body power: no differences } \\
\text { Agility: no differences } \\
\text { Anaerobic fitness: no differences } \\
\text { Inflammation: improved w/probiotic } \\
\text { Immunity: no differences } \\
\text { Body composition: no differences }\end{array}$ \\
\hline West et al. (42) & $\begin{array}{l}99(35) \text { cyclists } \\
35 \pm 9 \text { years }\end{array}$ & $\mathrm{DB}, \mathrm{RCT}$ & $\begin{array}{l}\text { L. fermentum VR1-003 (PCC) } 1 \mathrm{x} \\
10^{9} \mathrm{CFU} / \mathrm{d} \text { vs. placebo }\end{array}$ & 11 weeks & $\begin{array}{l}\text { Aerobic fitness: no differences } \\
\text { Illness: improved w/probiotic in male, worsened } \\
\text { w/probiotic in females } \\
\text { Inflammation: improved with probiotic } \\
\text { Immunity: no differences }\end{array}$ \\
\hline
\end{tabular}

CFU, colony forming units; w/, with; /d, per day; DB, double-blind; $R C T$, randomized controlled trial.

${ }^{a}$ Mean $\pm S D$; and/or range.

${ }^{b}$ L. acidophilus CUL-60 $1 \times 10^{10} \mathrm{CFU} / \mathrm{d}$, L. acidophilus CUL-21 $1 \times 10^{10} \mathrm{CFU} / \mathrm{d}$, B. bifidum CUL-20 $9.5 \times 10^{9} \mathrm{CFU} / \mathrm{d}$, B. animalis subsp. lactis CUL-34 $5 \times 10^{8}$ CFU/d, $0.6 \mathrm{~g}$ fructooligosaccharide.

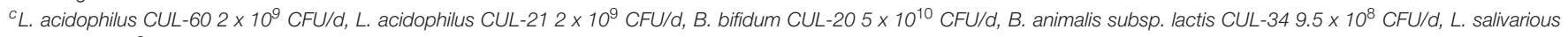
CUL-61 $5 \times 10^{9} \mathrm{CFU} / \mathrm{d}, 0.9 \mathrm{~g}$ L-glutamine.

B., Bifidobacterium; L., Lactobacillus. 
TABLE 2 | Probiotic influence on exercise-induced muscle damage in adults.

\begin{tabular}{|c|c|c|c|c|c|}
\hline References & Pop. $^{a}$ & Study design & Probiotic administration & Duration & Measures and results \\
\hline Gepner et al. (48) & $\begin{array}{l}17 \text { elite male } \\
\text { soldiers; } \\
20 \pm 2 \text { years }\end{array}$ & $\begin{array}{l}\mathrm{DB}, \mathrm{RCT} \\
\text { parallel }\end{array}$ & $\begin{array}{l}\text { B.coagulans } \mathrm{GBI}-30,6086 \text { (BC30; } \\
\left.1.0 \times 10^{9} \mathrm{CFU} / \mathrm{d}\right)+\mathrm{CaHMB} \text { vs. } \\
\text { CaHMB only during intense military } \\
\text { training }\end{array}$ & 40 days & $\begin{array}{l}\text { Serum inflammation markers: no differences } \\
\text { Serum muscle damage markers: no differences } \\
\text { Muscle integrity: improved in rectus femoris; no } \\
\text { differences in vastus lateralis }\end{array}$ \\
\hline Jager et al. (49) & $\begin{array}{l}29 \text { recreationally- } \\
\text { trained men; } \\
21 \pm 3 \text { years }\end{array}$ & $\begin{array}{l}\text { SB, not } \\
\text { random, } \\
\text { pre-post } \\
\text { design }\end{array}$ & $\begin{array}{l}\text { B. coagulans } \mathrm{GBI}-30,6086 \text { (BC30; } \\
\left.1.0 \times 10^{9} \mathrm{CFU} / \mathrm{d}\right)+ \text { casein vs. } \\
\text { casein alone prior to muscle } \\
\text { damaging exercise bout }\end{array}$ & 2 weeks & $\begin{array}{l}\text { Muscle soreness: improved w/ probiotic } 72 \mathrm{~h} \\
\text { post-exercise but not 24-48 h } \\
\text { Perceived recovery: improved w/probiotic } \\
\text { Serum muscle damage markers:trend for } \\
\text { improvement } \\
\text { Muscle swelling: no differences } \\
\text { Anaerobic power: trend for improvement } \\
\text { Lower-body power: no differences }\end{array}$ \\
\hline Jager et al. (50) & $\begin{array}{l}15 \text { resistance- } \\
\text { trained men; } \\
25 \pm 4 \text { years }\end{array}$ & $\begin{array}{l}\mathrm{DB}, \mathrm{RCT} \\
\text { crossover }\end{array}$ & $\begin{array}{l}\text { S. thermophilus FP4 }\left(5 \times 10^{9} \text { live }\right. \\
\text { cells/d), B. breve BR03 }\left(5 \times 10^{9} \text { live }\right. \\
\text { cells/d) vs. placebo prior to muscle } \\
\text { damaging exercise bout }\end{array}$ & 3 weeks & $\begin{array}{l}\text { Muscle soreness: no differences } \\
\text { Muscle swelling: no differences } \\
\text { Plasma inflammation markers: improved w/ } \\
\text { probiotic at rest but not after exercise } \\
\text { Plasma muscle damage markers: no differences } \\
\text { Peak torque: improved w/ probiotic } \\
\text { Range of motion: no differences }\end{array}$ \\
\hline
\end{tabular}

CaHMB, calcium $\beta$-hyroxy- $\beta$-methylbutyrate; CFU, colony forming units; w/, with; /d, per day; DB, double-blind; RCT, randomized-controlled trial; SB, single-blind.

a Study population. Age is mean $\pm S D$.

B., Bacillus; S., Streptococcus.

between improvements in markers of immune function or respiratory illness to performance outcomes. In most studies, performance was not measured or no performance advantage was observed. The specific reasons are unknown, but this highlights challenges and opportunities for evaluating probiotics in the context of immune function and performance moving forward. In particular, there are few related studies conducted in military training environments which are often characterized by multiple stressors that can potentially compromise immunity including climate, intense physical training, sleep deprivation, suboptimal nutrition, and psychological pressures. One of the few published studies included 47 male French Commando cadets who spent most of their time in "heavy physical activities" and were categorized as "sleep deprived" over a $\sim 4$ week training event (76). The authors concluded that benefits of probiotic supplementation in a multi-stressor environment relied mainly on capacity to prevent the spread of infection throughout the respiratory tract; however, no differences in URTI were observed between the intervention and placebo groups.

\section{Training Stress and Gastrointestinal Barrier Injury}

The lining of the GI tract is both a physical and immunological barrier, acting to deter the translocation of potentially harmful bacteria, toxins, and antigens into the systemic circulation while maintaining a selective permeability to nutrients (77, 78). GI barrier injury can lead to translocation of antigens such as bacterial LPS from the gut lumen into circulation. The resulting inflammation may contribute to GI distress and dysbiosis, adversely impact nutrient status, cognition and physical performance, and increase susceptibility to illness, infection and chronic disease (78-80). Of note, recent studies have reported increased GI permeability in military personnel during various training exercises in association with systemic inflammation, GI distress, increased blood brain barrier permeability, and changes in mood state (81-83). Those observations have stimulated interest in identifying interventions to prevent GI injury and mitigate increases in GI permeability within military environments.

To date, few studies have examined the efficacy of probiotics for mitigating GI barrier injury in healthy adults experiencing acute GI injury (Table 4). Those that have used different methods for inducing GI barrier injury included both prolonged moderate-to-high intensity exercise in various environments and non-steroidal anti-inflammatory drug (NSAID) ingestion. Of note, both stressors are common in military personnel $(44,86)$. Some, but not all, of these studies have reported beneficial effects of probiotic supplementation on GI permeability or other markers of GI barrier damage [Table 4; $(39,64,69,72,76,85)$ ]. Notably, the majority of studies reporting beneficial effects have used multi-strain formulations. For example, Lamprecht et al. reported that 14-week supplementation with a multi-strain probiotic preparation reduced fecal zonulin (an indicator of intestinal barrier permeability) concentrations, and attenuated post-exercise increases in some, but not all, markers of inflammation and oxidative stress in physically active men (64). Similarly, Roberts et al. reported that a multi-strain formulation prevented increases in intestinal permeability and reduced circulating endotoxin concentrations during recovery from a triathlon (72). In contrast, studies using single-strains generally have not reported beneficial effects. Rather, in one study, plasma endotoxin concentrations were elevated for $24 \mathrm{~h}$ following strenuous exercise in the heat following 7 days of administration of $L$. casei (unknown strain) relative to placebo, suggesting a detrimental effect (69). Of note, Lactobacillus spp. do not produce LPS, thus the increased endotoxin concentrations 
TABLE 3 | Impact of probiotics on Gl and respiratory immunity in adults.

\begin{tabular}{|c|c|c|c|c|c|c|c|}
\hline References & Pop. $^{a}$ & Study design & Probiotic administration & Duration & $\begin{array}{l}\text { Gl or respiratory } \\
\text { symptoms }\end{array}$ & Biochemistries & Performance outcome \\
\hline Clancy et al. (57) & $\begin{array}{l}17 \text { male \& } 10 \text { female } \\
\text { recreational athletes; } \\
16-40 \text { years }\end{array}$ & PPI & $\begin{array}{l}2 \times 10^{10} \mathrm{CFU} / \mathrm{d} \text { L. acidophilus } \\
\text { LAFTI L10 }\end{array}$ & 4weeks & $\begin{array}{l}\text { Fatigued athletes present } \\
\text { more episodes of URIs/year } \\
\text { and lost more activities to } \\
\text { illness }\end{array}$ & $\begin{array}{l}\text { Fatigued athletes: increased } \\
\text { IFN-gamma production by CD4 } \\
\text { cells Non- fatigued athletes: } \\
\text { increased salivary IFN-gamma }\end{array}$ & $\begin{array}{l}\text { No performance comparison } \\
\text { made between treatment groups. }\end{array}$ \\
\hline $\begin{array}{l}\text { Moreira et al. } \\
\text { (58) }\end{array}$ & $\begin{array}{l}123 \text { male \& } 16 \text { female } \\
\text { trained marathon } \\
\text { runners; } 39 \pm 9 \text { years }\end{array}$ & $\begin{array}{l}\mathrm{DB}, \mathrm{PC}, \mathrm{RCT} \\
\text { parallel }\end{array}$ & $\begin{array}{l}\text { Milk based L. rhamnosus GG } \\
\text { (LGG). } 3 \times 10^{8} \mathrm{CFU} / \mathrm{mL} \text {. } \\
\text { Participants drank } 130 \mathrm{~mL} / \text { day }\end{array}$ & 3 monthes & $\begin{array}{l}\text { No substantial difference in } \\
\text { symptoms of atopy or } \\
\text { asthma. }\end{array}$ & No difference between groups & $\begin{array}{l}\text { No significant difference in } \\
\text { marathon completion time } \\
\text { between the treatment groups. }\end{array}$ \\
\hline Tiollier et al. (59) & $\begin{array}{l}47 \text { trained French } \\
\text { Army cadets; } 21 \pm \\
0.4 \text { years }\end{array}$ & $\begin{array}{l}\mathrm{DB}, \mathrm{PC}, \mathrm{RCT} \\
\text { parallel }\end{array}$ & $\begin{array}{l}\text { Milk fermented by L. casei strain } \\
\text { DN-114 } 001\end{array}$ & $\begin{array}{l}3 \text { weeks }+ \\
5 \text { days }\end{array}$ & $\begin{array}{l}\text { No difference between } \\
\text { groups on ERTI in } \\
\text { incidence. }\end{array}$ & $\begin{array}{l}\text { Prevented the reduction of } \\
\text { salivary IgA after training. Immune } \\
\text { cells did not differ between } \\
\text { groups. } \\
\text { DHEA-S increased in probiotics } \\
\text { group. Cortisol and prolactin did } \\
\text { not change. }\end{array}$ & $\begin{array}{l}\text { No performance comparisons } \\
\text { made. }\end{array}$ \\
\hline $\begin{array}{l}\text { Kekkonen et al. } \\
(60)\end{array}$ & $\begin{array}{l}123 \text { male } \& 16 \text { female } \\
\text { trained marathon } \\
\text { runners; } 39 \pm 9 \text { years }\end{array}$ & $\begin{array}{l}\mathrm{DB}, \mathrm{PC}, \mathrm{RCT} \\
\text { parallel }\end{array}$ & $\begin{array}{l}\text { Milk based L. rhamnosus GG } \\
\text { (LGG). } 3 \times 10^{8} \mathrm{CFU} / \mathrm{mL} \text {. } \\
\text { Participants drank } 130 \mathrm{~mL} / \text { day }\end{array}$ & 3 months & $\begin{array}{l}\text { Decreased number (33\%) } \\
\text { and duration (57\%) of Gl } \\
\text { symptoms } 2 \text { weeks after } \\
\text { marathon, but no effects } \\
\text { related to URS incidence, } \\
\text { compared with placebo }\end{array}$ & $\begin{array}{l}\text { Hematological parameters within } \\
\text { reference range for both groups. }\end{array}$ & $\begin{array}{l}\text { No significant difference in } \\
\text { marathon completion time } \\
\text { between the treatment groups. }\end{array}$ \\
\hline Cox et al. (34) & $\begin{array}{l}20 \text { elite male runners; } \\
20-34 \text { years }\end{array}$ & $\mathrm{DB}, \mathrm{PC}$ & $\begin{array}{l}\text { Capsules containing L. fermentum. } \\
\text { VRI-003 (PCC) } 12 \times 10^{9} \mathrm{CFU} / \mathrm{d}\end{array}$ & 4 weeks & $\begin{array}{l}\text { Reduction in number (50\%) } \\
\text { of days with respiratory } \\
\text { illness symptoms } \\
\text { (self-reported) }\end{array}$ & $\begin{array}{l}\text { Modest increase in salivary IgA } \\
\text { and IgA1, and IFN- } \gamma \text {. No change } \\
\text { in IL- } 4 \text { and IL-12. }\end{array}$ & $\begin{array}{l}\text { No substantial changes in running } \\
\text { performance measures }\end{array}$ \\
\hline West et al. (42) & $\begin{array}{l}64 \text { male } 35 \pm 9 \text { years } \\
\& 35 \text { female } 36 \pm 9 \\
\text { years elite competitive } \\
\text { cyclists }\end{array}$ & $\begin{array}{l}\mathrm{DB}, \mathrm{PC}, \mathrm{RCT} \\
\text { parallel }\end{array}$ & $\begin{array}{l}\text { One capsule per day containing } L \text {. } \\
\text { fermentum(PCC) } 1 \times 10^{9} \mathrm{CFU} / \mathrm{d}\end{array}$ & 11 weeks & $\begin{array}{l}\text { Increase in mild Gl and } \\
\text { lower respiratory symptoms } \\
\text { compared to placebo. }\end{array}$ & $\begin{array}{l}\text { Reduced perturbations in } \\
\text { anti-inflammatory and } \\
\text { pro-inflammatory cytokines } \\
\text { (IL-1RA, IL-6, IL-8, IL-10, } \\
\text { GM-CSF, IFN- } \gamma \text {, TNF- } \alpha \text { ) in } \\
\text { probiotic group. }\end{array}$ & $\begin{array}{l}\text { No difference between groups in } \\
\text { performance tests (cycle } \\
\text { ergometry, } \mathrm{VO}_{2} \text { max) or exercise } \\
\text { duration. }\end{array}$ \\
\hline $\begin{array}{l}\text { Martarelli et al. } \\
\text { (61) }\end{array}$ & $\begin{array}{l}24 \text { male recreational } \\
\text { athletes; } 32 \pm 6 \text { years }\end{array}$ & PC, RCT, parallel & $\begin{array}{l}\text { Powdered mixtures of the } 2 \\
\text { probiotic strains }(1: 1 \text { L. rhamnosus } \\
\text { IMC } 501 \text { and Lactobacillus } \\
\text { paracasei IMC 502; } \sim 10 \times 10^{9} \\
\text { CFU/d) }\end{array}$ & 4 weeks & NR & $\begin{array}{l}\text { Increased plasma biological } \\
\text { antioxidant potential in probiotic } \\
\text { group. }\end{array}$ & $\begin{array}{l}\text { No performance comparisons } \\
\text { made between groups. }\end{array}$ \\
\hline $\begin{array}{l}\text { Gleeson et al. } \\
\text { (62) }\end{array}$ & $\begin{array}{l}54 \text { male \& } 30 \text { female } \\
\text { trained endurance } \\
\text { athletes; } 27 \pm 11.6 \\
\text { years }\end{array}$ & $\begin{array}{l}\mathrm{DB}, \mathrm{PC}, \mathrm{RCT} \\
\text { parallel }\end{array}$ & $\begin{array}{l}\text { Fermented milk containing } L \text {. casei } \\
\text { Shirota } 6.5 \times 10^{9} \mathrm{CFU} 2 \text { times per } \\
\text { day }\end{array}$ & 16 weeks & $\begin{array}{l}\text { Placebo group had } 36 \% \\
\text { more URS and higher URTI } \\
\text { episodes compared with } \\
\text { probiotic group (1.2 vs. 2.1). } \\
\text { Severity and duration of } \\
\text { symptoms were not } \\
\text { significantly different. }\end{array}$ & $\begin{array}{l}\text { Salivary IgA concentration was } \\
\text { higher after } 8 \text { and } 16 \text { weeks } \\
\text { compared to placebo. No } \\
\text { difference with IgG, IgM, or total } \\
\text { immunoglobulin. }\end{array}$ & $\begin{array}{l}\text { No performance comparisons } \\
\text { made between groups. }\end{array}$ \\
\hline
\end{tabular}


TABLE 3 | Continued

\begin{tabular}{|c|c|c|c|c|c|c|c|}
\hline References & Pop. $^{a}$ & Study design & Probiotic administration & Duration & $\begin{array}{l}\text { GI or respiratory } \\
\text { symptoms }\end{array}$ & Biochemistries & Performance outcome \\
\hline $\begin{array}{l}\text { Gleeson et al. } \\
(63)\end{array}$ & $\begin{array}{l}66 \text { trained endurance } \\
\text { athletes; } 19-28 \text { years }\end{array}$ & $\begin{array}{l}\text { DB, PC, RCT, } \\
\text { parallel }\end{array}$ & $\begin{array}{l}\text { Sachets containing L. salivarius* }{ }^{*} 2 \\
\times 10^{10} \mathrm{CFU} / \mathrm{d}\end{array}$ & 16 weeks & $\begin{array}{l}\text { No difference in URS } \\
\text { duration between groups, } \\
\text { no substantial difference in } \\
\text { frequency, duration, or } \\
\text { severity or URTI. }\end{array}$ & $\begin{array}{l}\text { No difference in salivary IgA } \\
\text { between groups. Probiotic group } \\
\text { increased lymphocyte totals, no } \\
\text { differences in other blood immune } \\
\text { cells. }\end{array}$ & $\begin{array}{l}\text { No performance comparisons } \\
\text { made between groups. }\end{array}$ \\
\hline $\begin{array}{l}\text { Lamprecht et al. } \\
\text { (64) }\end{array}$ & $\begin{array}{l}23 \text { male trained } \\
\text { athletes; } 38 \pm 5 \text { years }\end{array}$ & $\begin{array}{l}\text { DB, PC, RCT, } \\
\text { parallel }\end{array}$ & $\begin{array}{l}\text { Sachets containing B. bifidum W23 } \\
+ \text { B. lactis W51 + E. faecium W54 } \\
+ \text { L. acidophilus W22 + L. brevis } \\
\text { W63 }+ \text { L. lactis W58, } 1 \times 10^{10} \\
\text { CFU/d }\end{array}$ & 14 weeks & NR & $\begin{array}{l}\text { Reduced TNF concentration } \\
(25 \%) \text { at rest and post-exercise, } \\
\text { reduced exercise-induced protein } \\
\text { oxidation (8\%) compared to } \\
\text { placebo. No difference in IL-6 } \\
\text { production, or change in total } \\
\text { oxidation status of lipids and } \\
\text { malondialdehyde. }\end{array}$ & $\begin{array}{l}\text { No performance comparisons } \\
\text { made between groups. }\end{array}$ \\
\hline $\begin{array}{l}\text { Valimaki et al. } \\
\text { (65) }\end{array}$ & $\begin{array}{l}125 \text { male \& } 16 \text { female } \\
\text { trained runners; } 40 \\
\text { years (22-69) }\end{array}$ & $\begin{array}{l}\mathrm{DB}, \mathrm{PC}, \mathrm{RCT} \\
\text { parallel }\end{array}$ & $\begin{array}{l}\text { Milk based fruit drink with } \mathrm{L} \text {. } \\
\text { rhamnosus GG } 4 \times 10^{10} \mathrm{CFU} / \mathrm{d}\end{array}$ & 3 months & NR & $\begin{array}{l}\text { Oxidized LDL lipids increased by } \\
28 \% \text { and } 33 \% \text { during the } \\
\text { preparation period and decreased } \\
\text { by } 16 \% \text { and } 19 \% \text { during the } \\
\text { marathon run in the placebo and } \\
\text { probiotic groups, respectively. }\end{array}$ & $\begin{array}{l}\text { No performance comparisons } \\
\text { were made. }\end{array}$ \\
\hline West et al. (66) & $\begin{array}{l}241 \text { male } 35 \pm 12 \\
\text { years \& } 224 \text { female } 36 \\
\pm 12 \text { years trained } \\
\text { runners }\end{array}$ & $\begin{array}{l}\mathrm{DB}, \mathrm{PC}, \mathrm{RCT} \\
\text { parallel }\end{array}$ & $\begin{array}{l}\text { Sachets containing (i) B. animalis } \\
\text { subsp. lactis (BI-04), } 2.0 \times \\
10^{9} \mathrm{CFU} / \mathrm{d} \text { (ii) L. acidophilus NCFM } \\
\text { and B. animalis subsp. lactis Bi-07 } \\
\text { (NCFM \& Bi-07) } 5 \times 10^{9} \mathrm{CFU} / \mathrm{d}\end{array}$ & 164 days & $\begin{array}{l}\text { A reduction in URTI } \\
\text { episodes in probiotic } \\
\text { groups. Symptom severity } \\
\text { did not differ between } \\
\text { groups. }\end{array}$ & NR & $\begin{array}{l}\text { Significant decrease in activity } \\
\text { intensity but increase in activity } \\
\text { duration vs placebo. }\end{array}$ \\
\hline $\begin{array}{l}\text { Haywood et al. } \\
\text { (67) }\end{array}$ & $\begin{array}{l}30 \text { male elite rugby } \\
\text { players; 20-28 years }\end{array}$ & $\mathrm{PC}, \mathrm{RCT}$, parallel & $\begin{array}{l}\text { Capsules probiotics multi-species } \\
\text { (L. gasserit: } 2.6 \times 10^{12} \mathrm{CFU} / \mathrm{d}, \mathrm{B} \text {. } \\
\text { bifidum }^{*}: 0.2 \times 10^{12} \mathrm{CFU} / \mathrm{d} \text {, and } B \text {. } \\
\text { longum }^{*}: 0.2 \times 10^{12} \mathrm{CFU} / \mathrm{d} \text { ) }\end{array}$ & $\begin{array}{l}4 \text { weeks }+ \\
4 \text { weeks } \\
\text { washout }\end{array}$ & $\begin{array}{l}\text { Decreased incidence and } \\
\text { duration of URTI and GI } \\
\text { illness compared to } \\
\text { placebo. No difference in } \\
\text { symptom severity. }\end{array}$ & NR & $\begin{array}{l}\text { No performance comparisons } \\
\text { were made. }\end{array}$ \\
\hline Shing et al. (39) & $\begin{array}{l}10 \text { male trained } \\
\text { runners; } 27 \pm 2 \text { years }\end{array}$ & $\begin{array}{l}\mathrm{DB}, \mathrm{RCT}, \mathrm{PC} \text {, } \\
\text { cross-over }\end{array}$ &  & $\begin{array}{l}4 \text { weeks }+ \\
3 \text { weeks } \\
\text { washout }\end{array}$ & $\begin{array}{l}\text { Small reduction in } \\
\text { symptoms of Gl discomfort } \\
\text { compared to placebo }\end{array}$ & $\begin{array}{l}\text { A small-to-moderate reduction in } \\
\text { urine lactulose:rhamnose. } \\
\text { Significantly lower plasma LPS/GI } \\
\text { permeability in probiotic group. } \\
\text { No significant difference with IL-6, } \\
\text { IL-10, and IL-1ra compared to } \\
\text { placebo. No significant } \\
\text { differences with hematological } \\
\text { variables or urinary claudin-3 pre- } \\
\text { vs. post- exercise. }\end{array}$ & $\begin{array}{l}\text { Significant increase in running } \\
\text { time to fatigue in high } \\
\text { temperatures compared to } \\
\text { placebo }\end{array}$ \\
\hline $\begin{array}{l}\text { O'Brien et al. } \\
\text { (68) }\end{array}$ & $\begin{array}{l}67 \text { recreational but } \\
\text { untrained subjects; } \\
18-35 \text { years }\end{array}$ & PC, PPI & $\begin{array}{l}\text { Fermented kefir beverage } \\
\text { containing undefined } \\
\text { Lactobacillus } 1 \times 10^{9} \mathrm{CFU} / \text { serving, } \\
2 \text { servings/ week }\end{array}$ & 15 weeks & $N R$ & $\begin{array}{l}\text { Plasma c-reactive protein (CRP) } \\
\text { increased due to exercise, but no } \\
\text { difference due to probiotic } \\
\text { intervention. }\end{array}$ & $\begin{array}{l}\text { No performance comparison } \\
\text { made with respect to probiotic. }\end{array}$ \\
\hline
\end{tabular}


TABLE 3 | Continued

\begin{tabular}{|c|c|c|c|c|c|c|c|}
\hline References & Pop. ${ }^{a}$ & Study design & Probiotic administration & Duration & $\begin{array}{l}\text { Gl or respiratory } \\
\text { symptoms }\end{array}$ & Biochemistries & Performance outcome \\
\hline Gill et al. (69) & $\begin{array}{l}8 \text { male trained adults; } \\
26 \pm 6 \text { years }\end{array}$ & $\begin{array}{l}\text { B, RCT, PC, } \\
\text { cross-over }\end{array}$ & L. $\operatorname{casei}^{*}\left(1 \times 10^{11} \mathrm{CFU} / \mathrm{d}\right)$ & 1 weeks & NR & $\begin{array}{l}\text { No significant changes in resting } \\
\text { circulatory endotoxin } \\
\text { concentration or plasma cytokine } \\
\text { profile compared to placebo. } \\
\text { Relative to pre-EHS } \\
\text { concentrations, higher plasma } \\
\text { concentrations of endotoxin } \\
\text { TNF- } \alpha \text { were observed compared } \\
\text { to placebo. }\end{array}$ & $\begin{array}{l}\text { No performance comparisons } \\
\text { were made due to probiotic } \\
\text { intervention. }\end{array}$ \\
\hline $\begin{array}{l}\text { Gleeson et al. } \\
\text { (70) }\end{array}$ & $\begin{array}{l}156 \text { male, } 112 \text { female } \\
\text { recreational athletes; } \\
21 \pm 3 \text { years }\end{array}$ & $\begin{array}{l}\mathrm{DB}, \mathrm{PC}, \mathrm{RCT} \\
\text { parallel }\end{array}$ & $\begin{array}{l}\text { Fermented milk containing L. casei } \\
\text { Shirota } 6.5 \times 10^{9} \mathrm{CFU} / 2 \text { times per } \\
\text { day }\end{array}$ & 16 weeks & $\begin{array}{l}\text { No differences related to } \\
\text { URS, number of episodes, } \\
\text { total symptom score, or } \\
\text { episode duration. }\end{array}$ & $\begin{array}{l}\text { Decreased lgG-specific } \\
\text { antibodies for cytomegalovirus } \\
\text { (CMV) and Epstein-Barr virus } \\
\text { compared with baseline of } \\
\text { probiotic group. No differences in } \\
\text { immune cell counts. }\end{array}$ & $\begin{array}{l}\text { No performance comparisons } \\
\text { were made due to probiotic } \\
\text { intervention }\end{array}$ \\
\hline $\begin{array}{l}\text { Michalickova } \\
\text { et al. (71) }\end{array}$ & $\begin{array}{l}36 \text { male, } 14 \text { female } \\
\text { elite athletes; } 18-28 \\
\text { years }\end{array}$ & $\mathrm{DB}, \mathrm{PC}, \mathrm{RCT}$ & $\begin{array}{l}\text { Capsules containing L. helveticus } \\
\text { LaftiL10 } 2 \times 10^{10} \mathrm{CFU} / \mathrm{d}\end{array}$ & 14 weeks & $\begin{array}{l}\text { Decrease in URTI episode } \\
\text { duration and number of } \\
\text { symptoms compared to } \\
\text { placebo. No difference in } \\
\text { symptom severity and } \\
\text { incidence of URTI between } \\
\text { groups. }\end{array}$ & $\begin{array}{l}\text { No significant changes in } \\
\text { leukocyte abundance, TBF- } \beta \\
\text { serum levels, IL-10 from } \\
\text { peripheral blood mononuclear } \\
\text { cells (PBMCs), IFN- } \gamma \text { level from } \\
\text { PBMCs or viability/proliferation of } \\
\text { PBMCs upon antigen stimulation. } \\
\text { Group effect for CD4+/CD8+ } \\
\text { ratio was significant. }\end{array}$ & $\begin{array}{l}\text { No performance comparisons } \\
\text { were made due to probiotic } \\
\text { intervention }\end{array}$ \\
\hline $\begin{array}{l}\text { Roberts et al. } \\
\text { (72) }\end{array}$ & $\begin{array}{l}25 \text { male, } 5 \text { female } \\
\text { recreational triathletes; } \\
35 \pm 1 \text { years }\end{array}$ & $\mathrm{DB}, \mathrm{PC}, \mathrm{RCT}$ & $\begin{array}{l}\text { Capsule containing L. acidophilus } \\
\left(1 \times 10^{10} \mathrm{CFU} / \mathrm{d}, \mathrm{L} \text {. acidophilus }\right. \\
\mathrm{CUL}-60 \text { [NCIMB 30157] and } 1 \times \\
10^{10} \mathrm{CFU} / \mathrm{d} \text { L. acidophillus CUL-21 } \\
\text { [NCIMB } 30156]), 16.8 \mathrm{mg} / \mathrm{day} \mathrm{B} \text {. } \\
\text { bifidum and lactis }\left(9.5 \times 10^{9} \mathrm{CFU} / \mathrm{d}\right. \\
\text { B. bifidum CUL-20 [NCIMB30172] } \\
\text { and } 5 \times 10^{8} \mathrm{CFU} / \mathrm{d} \text { B. animalis } \\
\text { subsp. lactis CUL-34 [NCIMB } \\
30153]+55.8 \mathrm{mg} / \mathrm{d} \\
\text { fructooligosaccharide (FOS) with or } \\
\text { without antixodants }\end{array}$ & 12 weeks & $\begin{array}{l}\text { Gl symptom episodes were } \\
\text { lower in the probiotic + FOS } \\
\text { group at each month of } \\
\text { prerace training, and the } \\
\text { severity of Gl symptoms } \\
\text { was lower }\end{array}$ & $\begin{array}{l}\text { Reduction in plasma endotoxin } \\
\text { levels at pre-race and } 6 \text { days } \\
\text { post-race, as well as for lgG levels } \\
\text { recorded } 6 \text { d postrace. No } \\
\text { significant difference in Gl } \\
\text { permeability between groups } \\
\text { Lactose:Mannitol increased } \\
\text { marginally from baseline to } \\
\text { pre-race and } 6 \text { days post-race } \\
\text { with probiotic +antioxidant. }\end{array}$ & $\begin{array}{l}\text { Non-significant trend of faster } \\
\text { overall time to finish in probiotic } \\
\text { groups. }\end{array}$ \\
\hline $\begin{array}{l}\text { Strasser et al. } \\
\text { (73) }\end{array}$ & $\begin{array}{l}13 \text { male, } 16 \text { female } \\
\text { trained athletes; } \\
\text { 22-30 years }\end{array}$ & $\begin{array}{l}\mathrm{DB}, \mathrm{PC}, \mathrm{RCT} \\
\text { parallel }\end{array}$ & $\begin{array}{l}\text { Sachet containing } 1 \times 10^{10} \mathrm{CFU} \\
\text { multispecies B. bifidum W23 }+ \text { B. } \\
\text { lactisW51 + E. faeciumW54 }+ \text { L. } \\
\text { acidophilusW22 + L. brevis W63 + } \\
\text { L. lactisW58 }\end{array}$ & 3 months & $\begin{array}{l}\text { Incidence of URTI } \\
\text { decreased for both groups } \\
\text { over } 12 \text { weeks, yet fewer } \\
\text { probiotic treated subjects } \\
\text { had URTI after } 12 \text { weeks ( } 5 \\
\text { vs } 8 \text { ). }\end{array}$ & $\begin{array}{l}\text { After the acute exercise, probiotic } \\
\text { group lost less tryptophan vs. } \\
\text { placebo. Female participants had } \\
\text { higher degradation of tryptophan } \\
\text { compared with male participants. }\end{array}$ & $\begin{array}{l}\text { Significant increase in training } \\
\text { hours per week and decreased } \\
\text { resting energy expenditure } \\
\text { compared to placebo. }\end{array}$ \\
\hline
\end{tabular}


TABLE 3 | Continued

\begin{tabular}{|c|c|c|c|c|c|c|c|}
\hline References & Pop. $^{a}$ & Study design & Probiotic administration & Duration & $\begin{array}{l}\text { Gl or respiratory } \\
\text { symptoms }\end{array}$ & Biochemistries & Performance outcome \\
\hline $\begin{array}{l}\text { Marshall et al. } \\
\text { (38) }\end{array}$ & $\begin{array}{l}24 \text { male, } 6 \text { female } \\
\text { trained endurance } \\
\text { athletes; } 23-53 \text { years }\end{array}$ & RIM, parallel & $\begin{array}{l}\text { Capsules with or without glutamine } \\
\text { contained } 1 \times 10^{10} \mathrm{CFU} / \mathrm{d}, \mathrm{L} \text {. } \\
\text { acidophilus CUL-60 and } 1 \times 10^{10} \\
\mathrm{CFU} / \mathrm{d} \text { L. acidophillus CUL-21 } 16.8 \\
\mathrm{mg} / \mathrm{d} \text { B. bifidum }{ }^{*} \text { and lactis }(9.5 \times \\
10^{10} \mathrm{CFU} / \mathrm{d}, \mathrm{B} \text {. bifidum and } 0.5 \times \\
10^{10} \mathrm{CFU} / \mathrm{d} \text { B. animalis subspecies } \\
\text { lactis, and } 55.8 \mathrm{mg} / \mathrm{d} \\
\text { fructooligosaccharides (FOS) }\end{array}$ & 12 weeks & NR & $\begin{array}{l}\text { Blood eHSP72 was not different } \\
\text { between nutritional groups } \\
\text { (probiotic with or without } \\
\text { glutamine). }\end{array}$ & $\begin{array}{l}\text { Time to race completion was not } \\
\text { different between groups. }\end{array}$ \\
\hline $\begin{array}{l}\text { Michalickova } \\
\text { et al. (74) }\end{array}$ & $\begin{array}{l}22 \text { male elite athletes; } \\
20-24 \text { years }\end{array}$ & $\begin{array}{l}\mathrm{DB}, \mathrm{PC}, \mathrm{RCT} \\
\text { parallel }\end{array}$ & $\begin{array}{l}\text { Capsules containing L. helveticus } \\
\text { Lafti L10 }\left(2 \times 10^{10} \mathrm{CFU} / \mathrm{d}\right)\end{array}$ & 14 weeks & NR & $\begin{array}{l}\text { Decreased malondialdehyde } \\
\text { (MDA), superoxide dismutase } \\
\text { activity (SOD) serum paraoxonase } \\
\text { (PON1) compared to placebo. }\end{array}$ & $\begin{array}{l}\text { No performance comparisons } \\
\text { were made due to probiotic } \\
\text { administration. }\end{array}$ \\
\hline $\begin{array}{l}\text { Carbuhn et al. } \\
\text { (33) }\end{array}$ & $\begin{array}{l}20 \text { female elite } \\
\text { swimmers; 19-23 } \\
\text { years }\end{array}$ & $\begin{array}{l}\mathrm{DB}, \mathrm{PC}, \mathrm{RCT} \\
\text { parallel }\end{array}$ & $\begin{array}{l}\text { Capsules containing B. longum } \\
356241 \times 10^{9} \mathrm{CFU} / \mathrm{d}\end{array}$ & 6 weeks & $\begin{array}{l}\text { Mild improvement in } \\
\text { RESTQ52-sport weekly } \\
\text { self-regulation scores of } \\
\text { stress. No URTI or URS } \\
\text { measures. }\end{array}$ & $\begin{array}{l}\text { No difference in panel of systemic } \\
\text { inflammatory markers. Endotoxin } \\
\text { (LPS) and LPS-binding protein } \\
\text { (LPB) were not statistically } \\
\text { different between groups. Small } \\
\text { but significant decrease in the } \\
\text { systemic cytokine marker IL-1ra } \\
\text { within the probiotic group at } \\
\text { mid-training found. }\end{array}$ & $\begin{array}{l}\text { No significant difference between } \\
\text { supplemented groups }\end{array}$ \\
\hline $\begin{array}{l}\text { Komano et al. } \\
\text { (75) }\end{array}$ & $\begin{array}{l}51 \text { male recreational } \\
\text { athletes; } 19-21 \text { years }\end{array}$ & $\begin{array}{l}\mathrm{DB}, \mathrm{PC}, \mathrm{RCT} \\
\text { parallel }\end{array}$ & $\begin{array}{l}\text { Capsules containing heat killed } \\
\text { Lactococcus lactis JCM } 58051 \times \\
10^{11} \mathrm{CFU} / \mathrm{d}\end{array}$ & 13 days & $\begin{array}{l}\text { Significant decrease in } \\
\text { some respiratory symptoms } \\
\text { and cumulative days of } \\
\text { URTI, decreased fatigue } \\
\text { accumulation compared to } \\
\text { placebo. }\end{array}$ & $\begin{array}{l}\text { CD86 as maturation marker on } \\
\text { dendritic cell activity was } \\
\text { significantly increased in the } \\
\text { probiotic group at day } 14 .\end{array}$ & $\begin{array}{l}\text { No difference between training } \\
\text { time between groups. No } \\
\text { performance comparisons were } \\
\text { made due to probiotic } \\
\text { intervention. }\end{array}$ \\
\hline
\end{tabular}

Updated and adapted from AR 40-501 (2), Davies et al. (27), Pyne et al. (28), Coqueiro et al. (29). B, blinded; CFU, colony forming units; DB, double-blind; eHSP, extracellular heat shock protein; EHS, exertional heat stress Gl, gastrointestinal; NR, not reported; PC, placebo controlled, PPI, pre-post intervention; RCT, random controlled trial; RIM, randomized independent measures; TGF, transforming growth factor; URS, upper respiratory symptoms; URTI, upper respiratory tract infections, URI, upper respiratory infection.

${ }^{a}$ Mean $\pm S D$; and/or range.

*Strain not reported.

+ Species/strain not reported.

B., Bifidobacterium; C., Clostridium; E., Enterococcus; L., Lactobacillus, S., Streptococcus. 
TABLE 4 | Probiotic influence on markers of gastrointestinal barrier integrity in healthy adults.

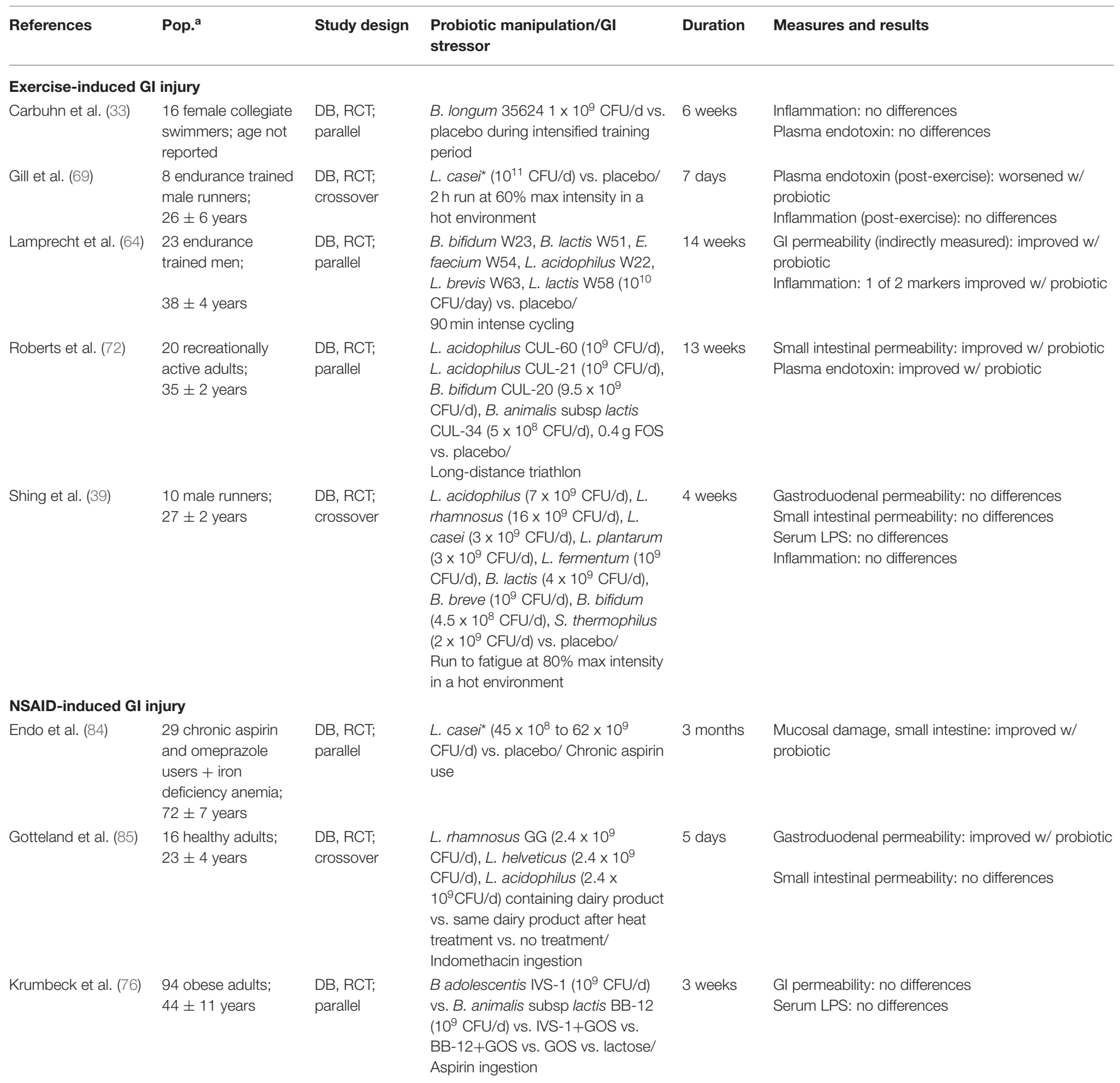

CFU, colony forming units; DB, double-blind; FOS, fructo-oligosaccharides; GI, gastrointestinal; GOS, galacto-oligosaccharide; LPS, lipopolysaccharide; NSAID, non-steroidal anti-inflammatory drug; $R C T$, randomized-controlled trial; $w /=$ with.

*Strain not reported.

${ }^{a}$ Mean $\pm S D$ and/or range.

B., Bifidobacterium; C., Clostridium; E., Enterococcus; L., Lactobacillus, S., Streptococcus.

did not reflect translocation of the ingested probiotic into circulation.

\section{Physical Domain Summary}

The ability of various different single-strain and multi-strain probiotic products to improve physical, primarily endurance, performance, often through effects on immunity, inflammation, and gut barrier integrity have been tested in athlete populations. Very few have been conducted in military populations. Within all of the physical performance related outcomes reviewed, some strains and strain-combinations have shown potential efficacy in single studies, but confirmatory studies are rare which precludes confident conclusions that any specific single- or multi-strain probiotic will benefit a particular outcome such as endurance 
TABLE 5 | Categorization of cognitive tasks.

\begin{tabular}{|c|c|}
\hline Cognitive domain & Cognitive tasks \\
\hline $\begin{array}{l}\text { Motor Speed and } \\
\text { Information Processing }\end{array}$ & $\begin{array}{l}\text { Cogstate Detection Test } \\
\text { Cogstate Groton Maze Chase Test } \\
\text { Cogstate Identification Test } \\
\text { Motor Screening Test }\end{array}$ \\
\hline Attention & Rapid Visual Information Processing Task \\
\hline Learning and Memory & $\begin{array}{l}\text { Cogstate Groton Maze Final Recall } \\
\text { Cogstate International Shopping List Test } \\
\text { Cogstate One Card Learning Test } \\
\text { Immediate and Delayed Recall } \\
\text { Paired Associates Learning } \\
\text { Wechsler Memory Scale }\end{array}$ \\
\hline Cognitive Control & $\begin{array}{l}\text { Attention Switching Task } \\
\text { Cogstate One Back Test } \\
\text { Digit Span Test } \\
\text { Emotional Stroop Test } \\
\text { Stroop Test } \\
\text { Verbal Learning Test }\end{array}$ \\
\hline
\end{tabular}

performance or exercise-induced muscle damage. This may indicate strain-specific effects, but could also reflect heterogeneity in the populations studied, dosages used, and duration of trials among other factors.

The majority of probiotic studies conducted in athlete populations have focused on immunity (32), and the incidence and severity of URTIs in particular. Again, some strains show benefit, while others do not, and confirmatory trials are rare. However, the multiple studies showing benefits coupled with meta-analyses suggesting favorable effects of probiotics on URTI incidence and severity in non-athlete populations support the need for clinical trials in military personnel, particularly during prolonged training events. Similarly, several, but not all, strains and strain combinations have shown beneficial effects on GI barrier injury during exercise. Those studies, coupled with evidence that certain probiotic supplements (i.e., Escherichia coli Nissle1917 and VSL\#3) improve symptomology in chronic GI diseases that are associated with barrier injury and increased permeability support the need for related research in military populations $(19,87,88)$. Thus, definitive recommendations for or against the use of certain single- and multi-strain probiotic formulations for favorable influence on physical performance and related outcomes in military personnel cannot be made at present. However, positive effects of some products in athlete and non-athlete population underscores the need for probiotic research focused on physical performance outcomes and mediators in military populations, and identifies potential candidates for testing.

\section{COGNITIVE AND PSYCHOLOGICAL HEALTH DOMAIN}

Emerging evidence suggests a bidirectional relationship between intestinal microbiota and human brain function, termed the "gutbrain axis." Intestinal microbes are thought to modulate this axis by altering the enteric nervous system and vagus nerve signaling, as well as immune function, and by producing compounds that enter systemic circulation and cross the blood brain barrier $(89,90)$. Probiotic intake has shown benefits in certain neurological disorders and may also ameliorate depressive and chronic fatigue syndrome, and anxiety symptoms (91, 92). In addition to emerging research on probiotics for psychological and neurological disorders, studies have also examined the influence on probiotic intake on cognitive function, mood, and emotional states in healthy individuals. Cognition, mood and emotion can be categorized into multiple sub-domains that are measured with a variety of different validated tests and scales (Tables 5, 7). Responses to these tests can vary along a continuum in healthy individuals, particularly in times of stress (93-95), and provide insight into effects of probiotics on cognition, mood and emotional state in healthy adults. Below we review studies assessing effects of probiotics within these sub-domains.

\section{Probiotic Effects on Cognitive Control in Healthy Adults}

Motor speed and information processing refers to the speed and accuracy of processing incoming information (96). Administration of single strains of Lactobacillus had marginal benefits in choice response time and social psychomotor performance but did not influence other measures of visuomotor speed, sensorimotor ability, or sustained attention (97, 98). Similarly, administration of $B$. longum did not influence sustained attention in another study (99). Together the findings provide little evidence of benefit of probiotic intake on motor speed, information processing, and attention, as summarized in Table 6.

Learning and memory is perhaps the most widely studied cognitive domain within the probiotic literature. Learning refers to a change in behavior resulting from experience, and memory refers to retaining and retrieving that information. In individuals experiencing moderate life stress, L. plantarum P8 intake improved episodic memory, compared to placebo, but had no effects on other aspects of learning and memory, such as visual learning and semantic memory (98). In another study, four to 12 weeks of multi-strain probiotic supplementation did not influence visual or verbal learning and memory across multiple tests $(97,99,101)$.

Episodic and working memory are cognitive domains most sensitive to decline with age, and thus older adults have been the primary focus in this area (104). Intake of a $L$. casei Shirota-containing milk drink worsened work memory compared to placebo after 20 days of consumption, and had no effect on episodic memory in one study of older adults (100). Twelve weeks of $L$. helveticus supplementation did not influence short- or long-term memory (102). Whether probiotics impact memory in healthy younger adults has not been studied.

Cognitive control, also called executive function, consists of mental set shifting (moving back and forth between tasks), information updating (integrating new information, also termed working memory), and inhibition (holding back a prepotent response) (105). In one comprehensive study of a multi-strain 
TABLE 6 | Probiotic influence on cognition in healthy individuals.

\begin{tabular}{|c|c|c|c|c|c|}
\hline References & Pop. $^{a}$ & Study design & Probiotic manipulation & Duration & Cognitive measures and results \\
\hline Allen et al. (99) & $\begin{array}{l}22 \text { healthy adults, } \\
25.5 \pm 1.2 \text { years }\end{array}$ & $\mathrm{DB}, \mathrm{RM}$ & B. longum 1714 strain vs. placebo & 4 weeks & $\begin{array}{l}\text { Learning: Probiotic improved } \\
\text { Sustained attention: No differences } \\
\text { Emotion recognition: No differences } \\
\text { Emotional Stroop: No differences }\end{array}$ \\
\hline Benton et al. (100) & $\begin{array}{l}126 \text { healthy adults, } \\
48-7961.8 \pm 7.3 \\
\text { years }\end{array}$ & $\mathrm{DB}, \mathrm{RCT}$ & $\begin{array}{l}65 \mathrm{~mL} \text { L. casei*-containing }(6.5 \times \\
\left.10^{9} \mathrm{CFU}\right) \text { vs. placebo milk }\end{array}$ & 20 days & $\begin{array}{l}\text { Short-term memory: Probiotic impaired after } 20 \text { (not } \\
\text { 10) days } \\
\text { Long-term memory: No differences } \\
\text { Verbal fluency: No differences } \\
\text { Intelligence: No differences }\end{array}$ \\
\hline Chong et al. (101) & $\begin{array}{l}\text { 111stressed adults, } \\
\text { 18-60 years }\end{array}$ & $\mathrm{DB}, \mathrm{RCT}$ & $\begin{array}{l}\text { L. plantarum DR7 }\left(1 \times 10^{9} \mathrm{CFU}\right) \text { vs. } \\
\text { placebo powder }\end{array}$ & 12 weeks & $\begin{array}{l}\text { Social Emotion Cognition: Probiotic improved speed } \\
\text { Verbal learning and memory: Probiotic improved } \\
\text { speed } \\
\text { Psychomotor performance: No differences } \\
\text { Attention: No differences } \\
\text { Visual learning and memory: No differences } \\
\text { Associate learning: No differences } \\
\text { Working memory: No differences } \\
\text { Executive function: No differences }\end{array}$ \\
\hline Chung et al. (102) & $\begin{array}{l}36 \text { healthy older } \\
\text { adults, } 60-7565.0 \\
\pm 1.1 \text { years }\end{array}$ & $\mathrm{DB}, \mathrm{RCT}$ & $\begin{array}{l}\text { L. helveticus IDCC3801 (500, } \\
1,000 \text {, vs. } 2,000 \text { mg) vs. placebo } \\
\text { capsules }\end{array}$ & 12 weeks & $\begin{array}{l}\text { Sustained Attention: 1,000 mg probiotic improved } \\
\text { Selective attention: } 500 \text { mg probiotic improved } \\
\text { Working memory: No differences } \\
\text { Short-term memory: No differences } \\
\text { Long-term memory: No differences }\end{array}$ \\
\hline Kelly et al. (97) & $\begin{array}{l}29 \text { healthy adults, } \\
20-3324.6 \pm 0.8 \\
\text { years }\end{array}$ & $\begin{array}{l}\mathrm{DB}, \mathrm{RCT} \\
\text { Cross-over }\end{array}$ & $\begin{array}{l}\text { L. rhamnosus JB- } 1\left(1 \times 10^{9} \mathrm{CFU}\right) \\
\text { vs. placebo capsules }\end{array}$ & 4 weeks & $\begin{array}{l}\text { Memory: No differences } \\
\text { Attention switching: No differences } \\
\text { Sustained Attention: No differences } \\
\text { Emotional interference: No differences } \\
\text { Emotion recognition: No differences }\end{array}$ \\
\hline Lew et al. (98) & $\begin{array}{l}103 \text { stressed adults, } \\
18-60 \text { years }\end{array}$ & $\mathrm{DB}, \mathrm{RCT}$ & $\begin{array}{l}\text { L. plantarum P8 }\left(2 \times 10^{10} \mathrm{CFU}\right) \text { vs. } \\
\text { placebo sachets }\end{array}$ & 12 weeks & $\begin{array}{l}\text { Social emotional cognition: Probiotic improved } \\
\text { speed } \\
\text { Memory: Probiotic improved } \\
\text { Target detection and identification: No differences }\end{array}$ \\
\hline Papalini et al. (103) & $\begin{array}{l}58 \text { healthy adults, } \\
18-40 \text { years }\end{array}$ & $\mathrm{DB}, \mathrm{RCT}$ & $\begin{array}{l}\text { Multispecies }{ }^{\mathrm{b}} \text { probiotic }\left(5 \times 10^{9}\right. \\
\text { CFU) vs. placebo powder }\end{array}$ & 4 weeks & $\begin{array}{l}\text { Emotional reactivity: No differences } \\
\text { Emotional interference: No differences } \\
\text { Selective attention: No differences } \\
\text { Pre vs. post stress working memory: Probiotic } \\
\text { improved }\end{array}$ \\
\hline
\end{tabular}

CFU, colony forming units; $D B$, double blind; RM, repeated; $M$, measures; $R C T$, randomized controlled trial. *Strain not reported.

${ }^{a}$ Mean $\pm S D$ and/or range ${ }^{b}$ B. bifidum W23, B. lactis W51, B. lactis W52, L. acidophilus W37, L. brevis W63, L. casei W56, L. salivarius W24, L. lactis W19, and L. lactis W58.

B, Bifidobacterium; L, Lactobacillus.

probiotic, supplementation did not influence selective attention, emotional interference or neural responses, but did improve working memory performance compared to placebo, following a stressor (103). These findings suggest that certain probiotics may ameliorate working memory deficits during stress (106, 107). In contrast, probiotics appear to exert fewer effects under non-stressful conditions. In support, of this observation single-strain Lactobacillus administration did not influence different measures of executive function or working memory in two studies $(97,101)$. Collectively, the existing evidence suggests that probiotic intake may exert benefits during stressful, rather than non-stressful, experiences. Further research should explore whether set-shifting and inhibition, in addition to information updating, are sensitive to probiotic improvements during stress.

\section{Probiotic Effects on Mood and Emotion, Depression, Anxiety, and Stress in Healthy Adults}

Emotions are episodic, specific to a triggering event (108). Moods are longer lasting affective states, not necessarily linked to a triggering event (109) (Table 7). In one study of older adults, consuming a $L$. casei Shirota-containing milk drink resulted in a reduction of feelings of depression, but not alteration in mood state (100). Similarly, in another study, individuals suffering moderate life stress at baseline experienced reduced feelings of stress and anxiety using one measurement scale but not another, following $L$. plantarum P8 supplementation (98). Correlation analyses of the cognitive findings reported above showed that social emotion cognition, and verbal learning and memory improved after 12 weeks of probiotic intake 
TABLE 7 | Categorization of mood and emotion scales.

\begin{tabular}{|c|c|}
\hline Affect & Assessment \\
\hline Depression & $\begin{array}{l}\text { Beck Depression Inventory } \\
\text { Depression, Anxiety and Stress Scale } \\
\text { Geriatric Depression Scale } \\
\text { Hospital Anxiety and Depression Scale } \\
\text { Leiden Index of Depression Sensitivity }\end{array}$ \\
\hline Anxiety & $\begin{array}{l}\text { Beck Anxiety Inventory } \\
\text { Depression, Anxiety and Stress Scale } \\
\text { Hospital Anxiety and Depression Scale } \\
\text { State Trait Anxiety Inventory }\end{array}$ \\
\hline Stress & $\begin{array}{l}\text { Depression, Anxiety and Stress Scale } \\
\text { Perceived Stress Scale }\end{array}$ \\
\hline Discrete Mood Scales & $\begin{array}{l}\text { Bond Lader Mood Scales } \\
\text { Profile of Mood States } \\
\text { Hopkins Symptom Checklist }\end{array}$ \\
\hline Emotion Regulation & $\begin{array}{l}\text { Coping Checklist } \\
\text { Primary Appraisal/Secondary Appraisal Scale }\end{array}$ \\
\hline
\end{tabular}

and were associated with reductions in stress and anxiety. In a study of individuals experiencing moderate life stress at baseline, probiotic administration ameliorated feelings of stress and anxiety, reduced cortisol and proinflammatory cytokine levels, and increased anti-inflammatory cytokine levels (101). The results point to a potential relationship between probioticinduced changes in mood, cognition, and the physiological stress response as seen in Table 8.

In individuals not characterized by elevated depression and stress, 30 days of supplementation with a multi-strain probiotic improved somatization, depression and angerhostility symptoms, depression, and anxiety, without impacting biomarkers of stress (111). Probiotic intake also reduced participants' reliance on self-blame as a coping strategy for negative experiences (111). Secondary analyses were performed in participants characterized by lower initial stress with probiotic intake also improving stress and obsessive compulsive and paranoid-ideation symptoms (111). In other studies, L. helveticus IDC3801-containing milk did not influence stress or depression in healthy older adults (102), and L. rhamnosus supplementation did not influence depression, anxiety, or stress, coping strategies to negative experiences, or emotional responses to an acute stressor in young adults (97). In healthy volunteers, daily intake of B. longum 1714 attenuated cortisol output and subjective anxiety in response to stress, and reduced daily reported stress. Resting electroencephalography (EEG) showed that B. longum increased frontal midline mobility, indicative of prefrontal cortex activity, and decreased $\mathrm{Cz}$-theta power, often associated with memory (99). The same strain did not influence emotional responses to a stressor involving social stress and exclusion in another study, but did influence brain activity as measured by magnetoencephalography (MEG) both during a resting state and following the social stressor. These results were interpreted to indicate that $B$. longum modulates neural oscillations in response to acute stress (116).

A number of studies have evaluated the influence of probiotic administration on cognitive reactivity to sad mood, in addition to sad moods themselves. Steenbergen and colleagues examined the influence of 4 weeks of administration of a multi-strain probiotic relative to placebo on cognitive reactivity to depressed mood (115). Probiotic administration reduced overall cognitive reactivity to depression, as well as cognitive reactivity to aggressive and ruminative thoughts specifically. Probiotic intake did not influence depression or anxiety (115). In other studies, 4 weeks of probiotic intake did not influence depression or cognitive reactivity to depressed mood (103), and 6 weeks of probiotic intake did not influence overall cognitive reactivity to depressed mood, but enhanced acceptance and coping of sad mood (110). Probiotic intake also reduced feelings of depression and anger, although not depression when assessed using an alternate measurement scale. Many of the studies described above also assessed the administration of probiotics on aspects of mood and emotion. The probiotics L. casei Shirota and L. plantarum P8 supplemented individually resulted in reduced negative mood; however, L. helveticus IDCC3801 and L. rhamnosus (JB-1) individually failed to produce similar outcomes (97, 98, 100, 102). Similarly, when multi-strain probiotics were administered, favorable effects were seen in some studies but not others $(111,115,117)$. Further, studies finding positive effects on some aspects of mood often failed to find effects on others, e.g., L. plantarum P8 reduced feelings of stress on one scale, but not on another scale, and did not reduce anxiety or depression (98). Of the 11 studies that examined whether probiotic administration benefits mood and emotion, seven found evidence of improvement (Table 8). Three studies found that probiotic administration reduced symptoms of depression and two studies found reduced cognitive reactivity to depressed $\operatorname{mood}(100,110,111,115)$. Four studies found that probiotic administration reduced symptoms of anxiety $(99,101,110,111)$. Four studies found that probiotic intake reduced perceived stress, two of those in populations characterized by moderate stress and two in populations characterized by normal to low stress $(98,99,101,112)$.

In summary, studies investigating the influence of probiotics on mood and emotion have selected a range of bacterial strains from the Lactobacillus or Bifidobacterium genera. Studies employing Lactobacillus strains found reduced negative mood with some species (casei and plantarum) but not others (helveticus and rhamnosus) (97, 98, 100102, 110). Similarly, two studies employing combinations of Lactobacillus and Bifidobacterium (L. helveticus and $B$. longum) found reduced negative mood while one (six strains of Lactobacillus and three strains of Bifidobacterium) did not $(103,111,115)$. Thus, the research to date does not point to a genus- or strain-specific effect of probiotics on mood. Nevertheless, the current evidence, suggests that probiotic intake may improve mood, particularly depressed, anxious, or stressed moods in healthy individuals free from mood disorders. Although the mechanism is not understood, it may involve reductions in physiological markers of stress and inflammation, such as cortisol and proinflammatory cytokines. 
TABLE 8 | Probiotic influence on mood in healthy individuals.

\begin{tabular}{|c|c|c|c|c|c|}
\hline References & Pop. $^{a}$ & Study design & Probiotic administration & Duration & Mood measures and results \\
\hline Allen et al. (99) & $\begin{array}{l}22 \text { healthy adults, } 25.5 \pm \\
1.2 \text { years }\end{array}$ & $\mathrm{DB}, \mathrm{RM}$ & B. longum 1714 strain vs. placebo & 4 weeks & $\begin{array}{l}\text { Stress: Probiotic reduced daily stress } \\
\text { Anxiety: Probiotic reduced }\end{array}$ \\
\hline Benton et al. (100) & $\begin{array}{l}126 \text { healthy adults, } 48-79 \\
61.8 \pm 7.3 \text { years }\end{array}$ & $\mathrm{DB}, \mathrm{RCT}$ & $\begin{array}{l}65 \mathrm{~mL} \text { L. casei*-containing }(6.5 \times \\
\left.10^{9} \mathrm{CFU}\right) \text { vs. placebo milk }\end{array}$ & $\sim 3$ weeks & $\begin{array}{l}\text { Elated/depressed: Probiotic reduced depression in } \\
\text { lowest baseline tertile for depression only } \\
\text { Energetic/ tired, clearheaded/muddled, } \\
\text { composed/anxious, confident/ unsure, } \\
\text { agreeable/angry: No differences }\end{array}$ \\
\hline Chong et al. (101) & $\begin{array}{l}111 \text { stressed adults, } \\
18-60 \text { years }\end{array}$ & $\mathrm{DB}, \mathrm{RCT}$ & $\begin{array}{l}\text { L. plantarum DR7 }\left(1 \times 10^{9} \mathrm{CFU}\right) \text { vs. } \\
\text { placebo powder }\end{array}$ & 12 weeks & $\begin{array}{l}\text { Stress: Probiotic reduced } \\
\text { Anxiety: Probiotic reduced } \\
\text { Depression: No differences }\end{array}$ \\
\hline Chung et al. (102) & $\begin{array}{l}36 \text { healthy older adults, } \\
60-75 \text { years }\end{array}$ & $\mathrm{DB}, \mathrm{RCT}$ & $\begin{array}{l}\text { L. helveticus IDCC3801 (500, } \\
1,000, \text { vs. 2,000 mg) vs. placebo } \\
\text { capsules }\end{array}$ & 12 weeks & $\begin{array}{l}\text { Depression: No differences } \\
\text { Stress: No differences }\end{array}$ \\
\hline Kelly et al. (97) & $\begin{array}{l}29 \text { healthy adults, } 20-33 \\
24.6 \pm 0.8 \text { years }\end{array}$ & $\begin{array}{l}\mathrm{DB}, \mathrm{RCT} \\
\text { Cross-Over }\end{array}$ & $\begin{array}{l}\text { L. rhamnosus JB- } 1\left(1 \times 10^{9} \mathrm{CFU}\right) \\
\text { vs. placebo capsules }\end{array}$ & 4 weeks & $\begin{array}{l}\text { Depression: No differences } \\
\text { Anxiety: No differences } \\
\text { Stress: No differences }\end{array}$ \\
\hline Lew et al. (98) & $\begin{array}{l}103 \text { stressed adults, } \\
18-60 \text { years }\end{array}$ & $\mathrm{DB}, \mathrm{RCT}$ & $\begin{array}{l}\text { L. plantarum P8 }\left(2 \times 10^{10} \mathrm{CFU}\right) \text { vs. } \\
\text { placebo sachets }\end{array}$ & 12 weeks & $\begin{array}{l}\text { Stress [DASS]: Probiotic reduced } \\
\text { Anxiety: Probiotic reduced } \\
\text { Stress [PSS]: No differences } \\
\text { Depression: No differences }\end{array}$ \\
\hline Marotta et al. (110) & $\begin{array}{l}38 \text { healthy adults, } 19-33 \\
22.00 \pm 3.02 \text { years }\end{array}$ & $\mathrm{DB}, \mathrm{RCT}$ & $\begin{array}{l}\text { L. fermentum LF16, L. rhamnosus } \\
\text { LR06, L. plantarum LP01, and B. } \\
\text { longum BL04 }\left(4 \times 10^{9} \mathrm{CFU}\right) \mathrm{vs} \text {. } \\
\text { placebo powder }\end{array}$ & 6 weeks & $\begin{array}{l}\text { Depression Sensitivity: No differences total score; } \\
\text { Probiotic increased acceptance } \\
\text { Anxiety: No differences } \\
\text { Depression [BDI]: No differences } \\
\text { Depression [POMS]: Probiotic reduced } \\
\text { Anger/hostility: Probiotic reduced }\end{array}$ \\
\hline $\begin{array}{l}\text { Messaoudi et al. } \\
(111)\end{array}$ & $\begin{array}{l}55 \text { healthy adults, } 30-60 \\
\text { Probiotic: } 42.4 \pm 7.5 \text { years } \\
\text { Placebo: } 43.2 \pm 8.5 \text { years }\end{array}$ & $\mathrm{DB}, \mathrm{RCT}$ & $\begin{array}{l}\text { L. helveticus R0052 and B. longum } \\
\text { R0175 }\left(3 \times 10^{9} \mathrm{CFU}\right) \text { vs. placebo } \\
\text { stick }\end{array}$ & 4 weeks & $\begin{array}{l}\text { Anxiety: Probiotic reduced anxiety } \\
\text { Depression [HSCL-90]: Probiotic improved } \\
\text { depression } \\
\text { Global Psychopathology Severity: Probiotic reduced } \\
\text { severity } \\
\text { Somatization: Probiotic reduced somatization } \\
\text { Anger/hostility: Probiotic reduced anger/hostility } \\
\text { Depression [HADS-D]: No differences } \\
\text { Stress: No differences }\end{array}$ \\
\hline $\begin{array}{l}\text { Messaoudi et al. } \\
(112)^{b}\end{array}$ & $\begin{array}{l}25 \text { healthy adults, 30-60 } \\
\text { years }\end{array}$ & $\mathrm{DB}, \mathrm{RCT}$ & $\begin{array}{l}\text { L. helveticus R0052 and B. longum } \\
\left.\text { R0175 ( } 3 \times 10^{9} \mathrm{CFU}\right) \text { vs. placebo } \\
\text { stick }\end{array}$ & 4 weeks & $\begin{array}{l}\text { Stress: Probiotic reduced } \\
\text { Obsessive compulsive: Probiotic reduced } \\
\text { Anxiety: Probiotic reduced } \\
\text { Paranoid-ideation: Probiotic reduced }\end{array}$ \\
\hline Noorwali et al. $(113)^{\mathrm{c}}$ & $\begin{array}{l}60 \text { healthy adults, } 18-40 \\
\text { years }\end{array}$ & $\mathrm{DB}, \mathrm{RCT}$ & $\begin{array}{l}\text { L. acidophilus CUL60 and CUL21, } \\
\text { B. lactis CUL34, and B. bifidum } \\
\text { CUL20 vs. placebo capsules }\end{array}$ & 6 weeks & Anxiety: No differences \\
\hline Owen et al. $(114)^{\mathrm{b}}$ & $\begin{array}{l}50 \text { healthy adults, } 19-38 \\
32.2 \pm 3.8 \text { years }\end{array}$ & $\mathrm{DB}, \mathrm{RCT}$ & $\begin{array}{l}\text { L. acidophilus CUL60 and CUL21, } \\
\text { B. lactis CUL34, and } B \text {. } \\
\text { bifidum CUL } 20\left(2.5 \times 10^{10} \text { CFU) vs. }\right. \\
\text { placebo capsules }\end{array}$ & 6 weeks & $\begin{array}{l}\text { Anxiety: Probiotic reduced } \\
\text { Depression: No differences } \\
\text { Stress: No differences }\end{array}$ \\
\hline Papalini et al. (103) & $\begin{array}{l}58 \text { healthy adults, } 18-40 \\
\text { years }\end{array}$ & $\mathrm{DB}, \mathrm{RCT}$ & $\begin{array}{l}\text { Multispecies }{ }^{d} \text { probiotic }\left(5 \times 10^{9}\right. \\
\text { CFU) vs. placebo powder }\end{array}$ & 4 weeks & $\begin{array}{l}\text { Depression: No differences } \\
\text { Depression sensitivity: No differences }\end{array}$ \\
\hline $\begin{array}{l}\text { Steenbergen et al. } \\
(115)\end{array}$ & $\begin{array}{l}40 \text { healthy adults, } \\
\text { Probiotic: } 20.2 \pm 2.4 \mathrm{yr} \\
\text { Placebo: } 19.7 \pm 1.7 \mathrm{yr}\end{array}$ & $\mathrm{DB}, \mathrm{RCT}$ & $\begin{array}{l}\text { Multispecies probiotic }\left(>2.5 \times 10^{9}\right. \\
\text { CFU/g) vs. placebo powder }\end{array}$ & 4 weeks & $\begin{array}{l}\text { Cognitive reactivity to sad mood: Probiotic reduced } \\
\text { Aggression: Probiotic reduced } \\
\text { Rumination: Probiotic reduced } \\
\text { Depression: No differences } \\
\text { Anxiety: No differences }\end{array}$ \\
\hline Wang et al. (116) & $\begin{array}{l}40 \text { healthy adults, } 18-50 \\
\text { years }\end{array}$ & $\mathrm{DB}, \mathrm{RCT}$ & $\begin{array}{l}\text { B. longum } 1714\left(1 \times 10^{9} \mathrm{CFU}\right) \text { vs. } \\
\text { placebo powder }\end{array}$ & 4 weeks & $\begin{array}{l}\text { Distress: No differences } \\
\text { Mood: No differences } \\
\text { Exclusion perception: No differences }\end{array}$ \\
\hline
\end{tabular}



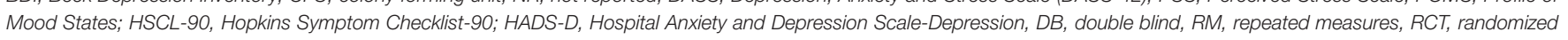
controlled trial, TB, triple blind.

*Strain not reported.

${ }^{a}$ Mean $\pm S D$ and/or range.

${ }^{b}$ Secondary analysis of Messaoudi et al. (111) in participants with lowest urinary free cortisol (UFC) levels at baseline.

${ }^{c}$ Conference Proceedings.

${ }^{d}$ B. bifidum W23, B. lactis W51, B. lactis W52, L. acidophilus W37, L. brevis W63, L. casei W56, L. salivarius W24, L. lactis (W19 and W58).

B, Bifidobacterium; L, Lactobacillus. 


\section{BRIDGING PHYSICAL AND PSYCHOLOGICAL DOMAINS: WOUND HEALING AND TBI}

Wound healing is an intricately orchestrated process comprised of the temporally sequenced, but overlapping stages of homeostasis, inflammation, proliferation, and remodeling $(118,119)$. Multiple factors common in military operational environments can disrupt wound healing. These include nonhygienic conditions, increased presence of pathogens and rate of infection, increased stress, immuno-compromise, heightened psychological stress, and suboptimal nutrition $(118,120,121)$. In support, it has been noted that deployment length correlates with level of psychological stress, which in turn, has been recognized to slow wound healing processes $(120,122)$. Impaired wound healing represents a significant healthcare burden for military populations, and current therapies are not always effective, underpinning an interest in developing novel approaches to improving wound care (119). Additionally, future military conflicts will likely require more field-based medical care before the wounded can be evacuated, further stimulating interest in identifying novel approaches to wound care that will decrease fatalities.

Probiotics have garnered some attention as potential adjuvants to standard wound care. Accumulating evidence from animal and in vitro studies suggest that these agents could improve wound healing through multiple mechanisms. Both topically and orally administered probiotics have been effective in animal studies, with, effects of oral probiotic therapies on wound healing thought to be mediated by interactions between the GI microbiota, enteric and central nervous systems, immune system, and skin microbiota (123). Whether these findings translate to human cohorts is unclear $(123,124)$. The largest body of clinical evidence derives from surgical care literature wherein several meta-analyses have examined whether probiotics and/or synbiotics (a combination of probiotics and prebiotics) reduce the incidence of post-operative infections, which impair healing of surgical wounds. In a recent metaanalysis of 28 studies including 2,511 patients undergoing GI surgery, perioperative synbiotic and probiotic administration reduced the likelihood of post-operative wound infection by 49 and $35 \%$, respectively (125). A separate meta-analysis including many of the same studies (31 total studies) and 2,952 patients undergoing elective abdominal surgery found that synbiotics and probiotics were more effective than placebo for reducing risk of post-operative surgical site infections ( 73 and $45 \%$ reduced risk, respectively) (126). Substantial heterogeneity across studies in the type, dose, timing of administration, and length of treatment (range: 3-25 days) was noted, although most studies used a combination of multiple probiotic strains $(125,126)$. Lactobacillus spp. comprised the most commonly investigated probiotics, and Bifidobacterium spp., Clostridium butryricum, Enterococcus faecalis T-110, Leuconostoc mesenteroides 77:1, Pediacoccuspentosaceus5-33:3, Streptococcus thermophilus, Streptococcus faecalis, and Saccharomyces boulardii were also used in one or more studies (126). In a study of older adults undergoing colorectal surgery, a probiotic cocktail containing $L$. acidophilus LA-5, L. plantarum, B. lactis BB-12, and S. boulardii administered 1day before and for 15 days after colorectal surgery significantly reduced the likelihood of post-operative wound infection (127). Few other clinical studies have examined the efficacy of probiotics for promoting wound healing [Table 9; (128-135)]. However, those studies have reported beneficial effects of orally-administered multi-strain probiotics on healing of chronic ulcers, and beneficial effects of topical single-strain products on healing of infected burns.

Several studies have examined effects of probiotics on skin barrier integrity, an integral component of wound healing that can be assessed by measuring transepidermal water loss (TEWL) (136). Because skin barrier damage increases water permeability through the skin, decreases in TEWL over time can be used to compare the efficacy of different interventions on wound healing (137). Studies using TEWL as an outcome have reported both positive and null results regarding the efficacy of probiotics for improving skin barrier integrity in individuals with various skin conditions ranging from sensitive skin to atopic dermatitis [Table 9; (129-131, 133)]. In one of the studies, a reduction in the colonization of the skin with Staphylococcus aureus, a common skin pathogen, was also observed using a topical lotion containing Vitreoscilla filiformis (129). However, to what extent those findings are applicable to wounds caused by trauma (e.g., laceration, burns) is uncertain.

Taken together, the current evidence base demonstrates a potential for probiotics, especially when paired with prebiotics (i.e., synbiotics), to reduce infection risk at post-operative wound sites relative to placebo which could improve post-operative wound healing. However, substantial heterogeneity in treatment strategies across studies currently prevents reaching conclusions on optimal strain selection, dosage, and timing of administration. Aside from studies conducted in patients undergoing elective surgery, there is currently little evidence to support or refute the use of probiotics or candidate probiotics for promoting wound healing in healthy adults (Table 9). Most, if not all, of the studies examining effects of probiotics on wound healing or related outcomes have been conducted in individuals with chronic health issues, and whether similar effects should be expected in healthy populations is unclear. Nonetheless, multiple plausible mechanisms by which probiotics could influence wound healing exist, and there is some support from both preclinical and clinical studies supporting efficacy $(123-126,138)$.

\section{Traumatic Brain Injury}

From 2000 to 2018, nearly 400,000 U.S. Armed Forces service members were diagnosed with traumatic brain injury (TBI) (139). Notably, TBI and comorbidities such as post-traumatic stress disorder (PTSD) have also been associated with gut microbiota dysbiosis in animal models, suggesting more work in humans is warranted (140-142). These associations have stimulated interest in examining the role of the gut microbiomegut-brain axis in the etiology and persistence of TBI-associated comorbidities, and in examining the effectiveness of probiotics as novel therapeutics in patients with a history of TBI (143-145). 
TABLE 9 | Probiotic influence on wound healing in adults.

\begin{tabular}{|c|c|c|c|c|c|}
\hline References & Pop. ${ }^{a}$ & Study design & Probiotic administration & Duration & Measures and results \\
\hline $\begin{array}{l}\text { Blanchet-Rethore } \\
\text { et al. (128) }\end{array}$ & $\begin{array}{l}21 \text { adults w/ atopic } \\
\text { dermatitis and } \\
\text { carrying } S . \text { aureus; } \\
33 \pm 12 \text { years }\end{array}$ & $\begin{array}{l}\text { NB; } \\
\text { non-random }\end{array}$ & $\begin{array}{l}\text { Heat-treated L. johnsonii NCC } 533 \\
\text { (HT La1) lotion } 0.3 \% \text { w/w twice daily } \\
\text { vs. untreated contralateral lesion }\end{array}$ & 3 weeks & S. aureus load: Improved w/probiotic \\
\hline Gueniche et al. (129) & $\begin{array}{l}75 \text { men/women w/ } \\
\text { atopic dermatitis; } \\
6-70 \text { years }\end{array}$ & $\begin{array}{l}\mathrm{DB}, \mathrm{RCT} \\
\text { parallel }\end{array}$ & $\begin{array}{l}\text { Lotion containing } 5 \% \text { V. filiformis* } \\
\text { lysate vs. placebo lotion }\end{array}$ & 30 days & $\begin{array}{l}\text { Lesion severity: Improved w/ probiotic } \\
\text { TEWL: no differences }\end{array}$ \\
\hline Gueniche et al. (130) & $\begin{array}{l}62 \text { women w/ } \\
\text { sensitive skin; } 32 \pm \\
12 \text { years }\end{array}$ & $\begin{array}{l}\mathrm{DB}, \mathrm{RCT} \\
\text { parallel }\end{array}$ & $\begin{array}{l}\text { L. paracasei NCC } 2461 \text { (ST11) } 1 \times \\
10^{10} \mathrm{CFU} / \mathrm{d} \text { vs. placebo }\end{array}$ & 8 weeks & TEWL: improved w/ probiotic \\
\hline Lee et al. (131) & $\begin{array}{l}110 \text { women w/ dry } \\
\text { skin; } 49 \pm 4 \text { years }\end{array}$ & $\begin{array}{l}\mathrm{DB}, \mathrm{RCT} \\
\text { parallel }\end{array}$ & $\begin{array}{l}\text { L. plantarum HY7714 } 1 \times 10^{10} \\
\text { CFU/d vs. placebo }\end{array}$ & 12 weeks & $\begin{array}{l}\text { TEWL, face: improved/ probiotic } \\
\text { TEWL, forearm: improved w/ probiotic } \\
\text { TEWL, hand: no differences }\end{array}$ \\
\hline Mohseni et al. (132) & $\begin{array}{l}60 \text { adults } w / \text { diabetic } \\
\text { foot ulcer; } 60 \pm 10 \\
\text { years }\end{array}$ & $\begin{array}{l}\mathrm{DB}, \mathrm{RCT} \\
\text { parallel }\end{array}$ & $\begin{array}{l}\text { L. acidophilus, L. casei, } L \text {. } \\
\text { fermentum, B. bifidum }{ }^{\star}\left(2 \times 10^{9}\right. \\
\text { CFU each/d) vs. placebo }\end{array}$ & 12 weeks & Wound healing: improved w/ probiotic \\
\hline Ogawa et al. (133) & $\begin{array}{l}118 \text { adults w/ } \\
\text { elevated TEWL; } 41 \\
\pm 8 \text { years }\end{array}$ & $\begin{array}{l}\mathrm{DB}, \mathrm{RCT} \\
\text { parallel }\end{array}$ & $\begin{array}{l}\text { Heat-killed L. brevis SBC8803 } 25 \text { or } \\
50 \text { mg/d vs. placebo }\end{array}$ & 12 weeks & $\begin{array}{l}\text { TEWL, forearm: no differences } \\
\text { TEWL, neck: no differences } \\
\text { TEWL, face: no differences }\end{array}$ \\
\hline Peral et al. (134) & $\begin{array}{l}80 \text { adult burn } \\
\text { patients; } 18-55 \text { year }\end{array}$ & $\begin{array}{l}\text { \#RCT; } \\
\text { parallel }\end{array}$ & $\begin{array}{l}\text { L. plantarum ATCC } 10241 \text { culture ( } 1 \\
\mathrm{~mL} / \mathrm{cm}^{2} \text { burn area vs. standard } \\
\text { care) }\end{array}$ & 10 days & $\begin{array}{l}\text { Wound healing: improved by probiotics in infected } \\
\text { 3rd degree burns, but not infected } 2 \text { nd degree } \\
\text { burns or non-infected 3rd degree burns. }\end{array}$ \\
\hline Peral et al. (135) & $\begin{array}{l}34 \text { adults w/ chronic } \\
\text { leg ulcers; } 40-70 \\
\text { years }\end{array}$ & $\begin{array}{l}\mathrm{NB} \\
\text { non-random; } \\
\text { pre-post trial }\end{array}$ & $\begin{array}{l}\text { L. plantarum ATCC } 10241 \text { culture } \\
\text { (no placebo) }\end{array}$ & $\begin{array}{l}10-30 \\
\text { days }\end{array}$ & $\begin{array}{l}\text { Wound healing: Total healing after } 30 \text { days in } 43 \% \\
\text { of diabetics and } 50 \% \text { of non-diabetics. }\end{array}$ \\
\hline
\end{tabular}

CFU, colony forming units; DB, double-blind; NB, not blinded; RCT, randomized controlled trial; TEWL, transepidermal water loss; w/ with.

a Population. Age is mean $\pm S D$ or range.

*Strain(s) not identified.

\#Blinding not described.

B, Bifidobacterium; L, Lactobacillus.

A recent systematic review by Brenner et al. identified only two published human studies examining probiotic interventions for treatment of TBI and/or PTSD (143). In one of the studies, brain trauma patients requiring enteral feeding had fewer infections, shorter stays in the intensive care unit, and fewer days on mechanical ventilation when receiving a diet supplemented with glutamine and Lactobacillus johnsonii ( $\mathrm{La}$ 1) compared to treatment with a standard diet (146). In a separate study, Tan et al. randomized patients with severe TBI receiving enteral nutrition to receive B. longum, L. bulgaricus, and S. thermophilus (strains not reported) in addition to standard treatment or standard treatment alone for 21 days (147). Patients receiving the probiotic intervention demonstrated an improved immune response, reduced inflammation, fewer infections, and shorter stays in the intensive care unit (147).

The results of those two studies are largely consistent with a recent meta-analysis of 30 trials which reported that using probiotics in the treatment of critical illness of varied etiologies, and $L$. plantarum-containing supplements in particular, was associated with a $20 \%$ reduction in infection risk (148). The TBI studies also provide preliminary evidence supporting beneficial effects of probiotics on clinical outcomes in the early stages of recovery from critical illness caused by severe brain injury. However, as was noted by Brenner et al., neither study included assessment of longer-term psychological outcomes following recovery from the initial brain injury (143). As such, the usefulness of probiotics in treating psychiatric comorbidities of TBI remains undetermined. Likewise, the extent to which the aforementioned findings relating to effects of probiotics on cognition and mood in healthy adults translate to improving cognitive and psychological function in individuals with a history of TBI is unclear. Nonetheless, the evidence derived from studies of probiotic use on cognitive and mood outcomes in adults without TBI does provide rationale for continued investigation into the efficacy of probiotics for treating cognitive and psychological symptoms in individuals with a history of TBI.

\section{GAPS AND CONSIDERATIONS}

Military personnel are required to maintain standards of physical and psychological performance throughout their military careers and in any environment. While many similarities exist between military personnel and "professional" athletes, professional athletes prepare and perform with a singular focus, whereas military personnel must incorporate a broader and more variable regime. Many similarities also exist between military personnel and healthy adults. However, military personnel often operate under combinations of stress and extreme environments rarely experienced by most adults. Recommendations for military personnel will therefore ideally be based on studies conducted with military personnel in the environments in which they 
TABLE 10 | Summary of probiotics used in Physical and Cognitive performance studies.

\begin{tabular}{|c|c|}
\hline Physical performance & Cognitive performance \\
\hline Bacillus coagulans GBI-30 & B. animalis subsp. lactis ${ }^{a}$ \\
\hline B. animalis subsp. lactis ${ }^{a}$ & B. bifidum CUL-20a \\
\hline B. bifidum $\mathrm{a}^{\mathrm{a}^{*}}$ & B. bifidum W23a \\
\hline B. bifidum W23a & B. lactis CUL-34 ${ }^{\mathrm{a}}$ \\
\hline B. breve* & B. lactis $\mathrm{W} 51^{\mathrm{a}}$ \\
\hline B. adolescentis IVS-1 & B. lactis $\mathrm{W} 52^{\mathrm{a}}$ \\
\hline B. infantis* & B. longum R0175a \\
\hline B. lactis* & L. acidophilus* \\
\hline B. lactis BB-12a & L. acidophilus CUL-21 a \\
\hline B. lactis $\mathrm{W} 51^{\mathrm{a}}$ & L. acidophilus CUL-60a \\
\hline B. longum ${ }^{*}$ & L. acidophilus W37ª \\
\hline B. longum 35624 & L. brevis $\mathrm{W} 63^{\mathrm{a}}$ \\
\hline B. subtilis DE110 & L. casei Shirota \\
\hline C. butryricum ${ }^{\star}$ & L. caseiW56 ${ }^{a}$ \\
\hline E. coli Nissle 1917 & L. helveticus IDCC3801ª \\
\hline E. faecalis T-110 & L. helveticus R0052 ${ }^{\mathrm{a}}$ \\
\hline Enterococcus faecium W54a & L. lactis W19a \\
\hline$\underline{\text { L. acidophilus }}{ }^{\star}$ & L. lactis W58 ${ }^{a}$ \\
\hline L. acidophilus CUL-21 ${ }^{a}$ & L. plantarum P8 \\
\hline L. acidophilus CUL-60ª & L. rhamnosus JB-1 \\
\hline L. acidophilus LA-5a & L. salivarius W24 ${ }^{\mathrm{a}}$ \\
\hline \multicolumn{2}{|l|}{ L. acidophilus W22a } \\
\hline \multicolumn{2}{|l|}{ L. brevis $\mathrm{W}^{6} 3^{\mathrm{a}}$} \\
\hline \multicolumn{2}{|l|}{ L. bulgaricus ${ }^{\mathrm{a}}$} \\
\hline \multicolumn{2}{|l|}{ L. casei* } \\
\hline \multicolumn{2}{|l|}{ L. dulbruecki subsp bulgaris } \\
\hline \multicolumn{2}{|l|}{ L. fermentum ${ }^{\mathrm{a}^{*}}$} \\
\hline \multicolumn{2}{|l|}{ L. fermentumVRI-003 } \\
\hline \multicolumn{2}{|l|}{ L. helveticus* } \\
\hline \multicolumn{2}{|l|}{ L. paracasei* } \\
\hline \multicolumn{2}{|l|}{ L. plantarum $^{\star}$} \\
\hline \multicolumn{2}{|l|}{ L. plantarum 299V } \\
\hline \multicolumn{2}{|l|}{ L. plantarum ATCC10241 } \\
\hline \multicolumn{2}{|l|}{ L. plantarum PS128 } \\
\hline \multicolumn{2}{|l|}{ L. plantarum WCFS1 } \\
\hline \multicolumn{2}{|l|}{ L. rhamnosus ${ }^{a}$} \\
\hline \multicolumn{2}{|l|}{ L. rhamnosus GG } \\
\hline \multicolumn{2}{|l|}{ Lactococcus lactis W58 ${ }^{a}$} \\
\hline \multicolumn{2}{|l|}{ Leuconostoc mesenteroides $77: 1$} \\
\hline \multicolumn{2}{|l|}{ P. pentasaceus 5-33:3 } \\
\hline \multicolumn{2}{|l|}{ S. boulardiia } \\
\hline \multicolumn{2}{|l|}{ Streptococcus faecalis* } \\
\hline Streptoccus thermophilus ${ }^{*}$ & \\
\hline
\end{tabular}

Probiotics administered both alone and as part of a cocktail;

a probiotics administered solely in a cocktail.

${ }^{\star}$ Strain not reported.

B, Bifidobacterium; C, Clostridium; E, Escherichia; L, Lactobacillus, S., Saccharomyces.

operate. Unfortunately, to date, few studies have examined effects of probiotics in military personnel $(48,59)$. However, it would be imprudent to not consider military applications of research conducted in other populations when evaluating potential applications of probiotics in military populations and identifying candidate strains for testing.

This narrative review has considered a growing evidence base that has assessed performance within physical, cognitive, and psychological domains following probiotic supplementation in healthy athletes and non-athletes (Table 10). In the course of the review, several knowledge gaps relevant to transitioning available evidence to military populations, and considerations for making recommendations for military populations were identified:

1) Studies do not always evaluate cognitive, psychological, and physical performance endpoints that are relevant for military training and performance, and generally do not incorporate measures assessing both performance domains. Multi-disciplinary studies are needed to address the complex interactions between physical and psychological stress imposed on military personnel.

2) Strain specificity is important for several, if not all, outcomes. However, studies reporting favorable effects of individual formulations, particularly within the physical performance domain, generally have not been reproduced. Confirmatory studies, especially those providing mechanistic insight, are needed to draw more definitive conclusions with respect to cause and effect.

3) Little consideration has been given to optimizing the timing, duration, and dose of probiotic administration. Studies examining these issues would provide a powerful approach to confirming the efficacy of promising formulations. Studies focused on optimizing the duration and dosing of supplementation are particularly relevant to military populations who may not receive much advanced notice before being placed in physically and psychologically stressing environments.

4) Little consideration has been given to how individual variability may contribute to probiotic effectiveness. Differences in sex, age, fitness level, gut microbiota composition, lifestyle habits, and environmental exposures may all influence interactions between a probiotic and host. Understanding these differences could conceivably lead to opportunities for personalized probiotic recommendations, or, more likely, recommendations for certain populations or environments.

5) Probiotic formulations are particularly relevant in certain military environments. Many probiotic strains require refrigeration which may not always be available to military personnel, and will not maintain viability under the extreme conditions required for military ration development and storage. One alternative approach to consider for future research is to consider prebiotics, inactivated microbes, and/or postbiotics which refers to metabolites and other compounds produced by microorganisms (149).

6) While probiotics have a demonstrated long history of safe use, the safety of novel candidate strains cannot be assumed. Indeed, caution is recommended when using probiotics in immunocompromised individuals and critical care patients (23). Safety and efficacy is required to be demonstrated 
for the targeted population. Further, the safety of dietary supplements is also not always known, and inaccurate claims, labels and/or unspecified product ingredients have been found on the market $(18,150)$. Research using off-the shelf products must verify what is actually in the product prior to testing.

7) With the exception of a few studies conducted in military cohorts, the physical and psychological stressors studied are not fully representative of what military personnel experience. Future studies in military populations should replicate, as much as possible, environmental and occupational stressors in order to fully elucidate any benefits of probiotic supplementation.

\section{FUTURE DIRECTIONS FOR MILITARY PROBIOTIC RESEARCH}

Adequately powered, double-blind, randomized controlled trials conducted in military personnel exposed to the range and combinations of stressors in which military populations operate will be the gold standard for determining probiotic application for military personnel. Studies should use well-defined probiotic strains, consider dose, timing of administration, and product formulations, and determine the impact of inter-individual differences and environmental exposures on probiotic-host interactions. To expedite transition, studies should leverage the research reviewed herein by attempting to replicate and extend favorable effects observed in athlete or other populations. Care should be taken not to generalize positive or null results to all strains, populations or environments. To the extent possible, these studies should also aim to glean mechanistic insight into host-probiotic interactions to better establish causal relationships.

\section{CONCLUSION}

A growing body of evidence has examined the effects of a range of probiotic products on physical and cognitive performance in healthy young adult populations, to include athlete populations (32). These studies are building on an evidence base that has demonstrated efficacy of probiotics in a variety of clinical applications ranging from treating symptoms of GI disorder to preventing post-operative infections. We

\section{REFERENCES}

1. AR 350-1 Army Training and Education. D.O.T. Army (2003).

2. AR 40-501 Standards of Medical Fitness. D.O.T. Army (2007).

3. FM 7-22 Army Physical Readiness Training. D.O.T. Army (2012).

4. Austin KG, Price LL, Mcgraw SM, Mclellan TM, Lieberman HR. Longitudinal trends in use of dietary supplements by U.S. Army personnel differ from those of civilians. Appl Physiol Nutr Metab. (2016) 41:1217-24. doi: 10.1139/apnm-2016-0296

5. Knapik JJ, Trone DW, Austin KG, Steelman RA, Farina EK, Lieberman HR. Prevalence, adverse events, and factors associated with dietary supplement and nutritional supplement use by US navy and marine corps personnel. $J$ Acad Nutr Diet. (2016) 116:1423-42. doi: 10.1016/j.jand.2016.02.015 conclude that there is currently not compelling evidence to demonstrate that probiotics globally improve human physical performance, cognition or mood in healthy adults, and military personnel in particular. As such, recommending probiotic use for military personnel is premature. Promising evidence for strain-specific effects, and perhaps more broad effects in the case of immune function, has been demonstrated in some studies. It should not be expected that all probiotics will have similar effects, or that individual probiotics will have favorable effects for all outcomes, within all populations, and across all environments. As such, the possibilities for research on different strain combinations, administered in different forms and doses to different populations in different environments are seemingly endless. While testing these combinations in military personnel would be ideal for translation of findings into specific recommendations, it is not conceivable. Therefore, to expedite transition, it is recommended that studies conducted in comparable civilian populations be used to inform design of confirmatory studies in military cohorts especially when plausible mechanistic evidence is available. Those studies must consider unique exposures and requirements of military personnel to include optimal product dosing, timing and formulation, and the effects of inter-individual and environmental variability.

\section{AUTHOR CONTRIBUTIONS}

JK, SL, and KR contributed to physical performance sections. GG contributed to cognitive sections. RA, JK, MG, KM, and JS contributed to introduction and consensus sections, manuscript content and organization. All authors contributed to literature review and manuscript editing.

\section{FUNDING}

RA was supported by Office of Naval Research.

\section{ACKNOWLEDGMENTS}

We would like to thank Dr. Linda Chrisey from Office of Naval Research for valuable comments during review of this manuscript. We would also like to thank Laura F. Kelly, CCDCSC G9 -Visual Communication/ Graphics, for graphic/figure design, creation and support.
6. Knapik JJ, Austin KG, Farina EK, Lieberman HR. Dietary supplement use in a large, representative sample of the US armed forces. J Acad Nutr Diet. (2018) 118:1370-88. doi: 10.1016/j.jand.2018.03.024

7. Hatch AM, Cole RE, Dichiara AJ, Mcgraw SM, Merrill EP, Wright AO, et al. Personality traits and occupational demands are linked to dietary supplement use in soldiers: a cross-sectional study of sensation seeking behaviors. Mil Med. (2019) 184:e253-62. doi: 10.1093/milmed/usy201

8. Reid G, Gadir AA, Dhir R. Probiotics: reiterating what they are and what they are not. Front Microbiol. (2019) 10:424. doi: 10.3389/fmicb.2019. 00424

9. Babio N, Becerra-Tomas N, Martinez-Gonzalez MA, Corella D, Estruch $\mathrm{R}$, Ros E, et al.Consumption of yogurt, low-fat milk, and other lowfat dairy products is associated with lower risk of metabolic syndrome 
incidence in an elderly mediterranean population. J Nutr. (2015) 145:230816. doi: $10.3945 /$ jn. 115.214593

10. Markowiak P, Slizewska K. Effects of probiotics, prebiotics, and synbiotics on human health. Nutrients. (2017) 9:1021. doi: 10.3390/nu9091021

11. Khalesi S, Bellissimo N, Vandelanotte C, Williams S, Stanley D, Irwin C. A review of probiotic supplementation in healthy adults: helpful or hype? Eur J Clin Nutr. (2019) 73:24-37. doi: 10.1038/s41430-018-0135-9

12. Hill C, Guarner F, Reid G, Gibson GR, Merenstein DJ, Pot B, et al. Expert consensus document. The International Scientific Association for Probiotics and Prebiotics consensus statement on the scope and appropriate use of the term probiotic. Nat Rev Gastroenterol Hepatol. (2014) 11:50614. doi: 10.1038/nrgastro.2014.66

13. Hao Q, Dong BR, Wu T. Probiotics for preventing acute upper respiratory tract infections. Cochrane Database Syst Rev. (2015) 9:CD006895. doi: 10.1002/14651858.CD006895.pub3

14. Wang Y, Li X, Ge T, Xiao Y, Liao Y, Cui Y, et al. Probiotics for prevention and treatment of respiratory tract infections in children: A systematic review and meta-analysis of randomized controlled trials. Medicine. (2016) 95:e4509. doi: 10.1097/MD.0000000000004509

15. Szilagyi A, Ishayek N. Lactose intolerance, dairy avoidance, treatment options. Nutrients. (2018) 10. doi: 10.3390/nu10121994

16. Hoffmann DE, Fraser CM, Palumbo F, Ravel J, Rowthorn V, Schwartz J. Probiotics: achieving a better regulatory fit. Food Drug Law J. (2014) 69:237-72, ii.

17. Buggio L, Somigliana E, Borghi A, Vercellini P. Probiotics and vaginal microecology: fact or fancy? BMC Women's Health. (2019) 19:25. doi: 10.1186/s12905-019-0723-4

18. De Simone C. The unregulated probiotic market. Clin Gastroenterol Hepatol. (2019) 17:809-17. doi: 10.1016/j.cgh.2018.01.018

19. Skokovic-Sunjic D. 2019 USA Clinical Guide to Probiotic Products: Indications, Dosage Formats, and Clinical Evidence to Date; Annual update (2019).

20. Sanders ME, Heimbach JT, Pot B, Tancredi DJ, Lenoir-Wijnkoop I, Lahteenmaki-Uutela A, et al. Health claims substantiation for probiotic and prebiotic products. Gut Microbes. (2011) 2:127-33. doi: 10.4161/gmic.2.3.16174

21. Merenstein D, Guzzi J, Sanders ME. More information needed on probiotic supplement product labels. J.Gen. Intern. Med. (2019) 34:27357. doi: 10.1007/s11606-019-05077-5

22. Dailey Z, Sanders ME, Merenstein D. Retail refrigerated probiotic foods and their association with evidence of health benefits. Benef Microbes. (2020) 11:131-3. doi: 10.3920/BM2019.0162

23. Sanders ME, Merenstein DJ, Ouwehand AC, Reid G, Salminen S, Cabana MD, et al. Probiotic use in at-risk populations. J Am Pharm Assoc. (2016) 56:680-6. doi: 10.1016/j.japh.2016.07.001

24. Sanders ME, Merenstein D, Merrifield CA, Hutkins R. Probiotics for human use. Nutr Bull. (2018) 43:212-25. doi: 10.1111/nbu.12334

25. Smith TJ, Margolis LM, Young AJ. Should military dining facilities offer and promote consumption of probiotic-containing foods? Milit Med. (2010) 175:770-83. doi: 10.7205/MILMED-D-10-00024

26. Egan B, Zierath JR. Exercise metabolism and the molecular regulation of skeletal muscle adaptation. Cell Metab. (2013) 17:162-84. doi: 10.1016/j.cmet.2012.12.012

27. Davies KJ, Packer L, Brooks GA. Biochemical adaptation of mitochondria, muscle, and whole-animal respiration to endurance training. Arch Biochem Biophys. (1981) 209:539-54. doi: 10.1016/0003-9861(81)90312-X

28. Pyne DB, West NP, Cox AJ, Cripps AW. Probiotics supplementation for athletes - clinical and physiological effects. Eur J Sport Sci. (2015) 15:6372. doi: 10.1080/17461391.2014.971879

29. Coqueiro AY, De Oliveira Garcia AB, Rogero MM, Tirapegui J. Probiotic supplementation in sports and physical exercise: Does it present any ergogenic effect? Nutr Health. (2017) 23:239-49. doi: 10.1177/0260106017721000

30. Mach N, Fuster-Botella D. Endurance exercise and gut microbiota: a review. J Sport Health Sci. (2017) 6:179-97. doi: 10.1016/j.jshs.2016.05.001

31. Leite GSF, Resende AS, West NP, Lancha AH Jr. Probiotics and sports: a new magic bullet? Nutrition. (2019) 60:152-60. doi: 10.1016/j.nut.2018.09.023
32. Jäger R, Mohr AE, Carpenter KC, Kerksick CM, Purpura M, Moussa A, et al. International society of sports nutrition position stand: probiotics. J Int Soc Sports Nutr. (2019) 16:62. doi: 10.1186/s12970-019-0329-0

33. Carbuhn AF, Reynolds SM, Campbell CW, Bradford LA, Deckert JA, Kreutzer A, et al. Effects of probiotic (Bifidobacterium longum 35624) supplementation on exercise performance, immune modulation, and cognitive outlook in division I female swimmers. Sports. (2018) 6:E116. doi: 10.3390/sports6040116

34. Cox AJ, Pyne DB, Saunders PU, Fricker PA. Oral administration of the probiotic Lactobacillus fermentum VRI-003 and mucosal immunity in endurance athletes. $\mathrm{Br} J$ Sports Med. (2010) 44:222-6. doi: 10.1136/bjsm.2007.044628

35. Huang WC, Hsu YJ, Li H, Kan NW, Chen YM, Lin JS, et al. Effect of Lactobacillus plantarum TWK10 on improving endurance performance in humans. Chin J Physiol. (2018) 61:163-70. doi: 10.4077/CJP.2018.BAH587

36. Huang WC, Wei CC, Huang CC, Chen WL, Huang HY. The beneficial effects of Lactobacillus plantarum PS128 on high-intensity, exercise-induced oxidative stress, inflammation, and performance in triathletes. Nutrients. (2019) 11:E353. doi: 10.3390/nu11020353

37. Ibrahim NS, Muhamad AS, Ooi FK, Meor-Osman J, Chen CK. The effects of combined probiotic ingestion and circuit training on muscular strength and power and cytokine responses in young males. Appl Physiol Nutr Metab. (2018) 43:180-6. doi: 10.1139/apnm-2017-0464

38. Marshall H, Chrismas BCR, Suckling CA, Roberts JD, Foster J, Taylor L. Chronic probiotic supplementation with or without glutamine does not influence the eHsp72 response to a multi-day ultra-endurance exercise event. Appl Physiol Nutr Metab. (2017) 42:876-83. doi: 10.1139/apnm-201 7-0131

39. Shing CM, Peake JM, Lim CL, Briskey D, Walsh NP, Fortes MB, et al. Effects of probiotics supplementation on gastrointestinal permeability, inflammation and exercise performance in the heat. Eur J Appl Physiol. (2014) 114:93-103. doi: 10.1007/s00421-013-2748-y

40. Toohey JC, Townsend JR, Johnson SB, Toy AM, Vantrease WC, Bender D, et al. Effects of probiotic (Bacillus subtilis) supplementation during offseason resistance training in female division I athletes. J Strength Cond Res. (2018). doi: 10.1519/JSC.0000000000002675

41. Townsend JR, Bender D, Vantrease WC, Sapp PA, Toy AM, Woods CA, et al. Effects of probiotic (Bacillus subtilis DE111) supplementation on immune function, hormonal status, and physical performance in division I baseball players. Sports. (2018) 6:E70. doi: 10.3390/sports6030070

42. West NP, Pyne DB, Cripps AW, Hopkins WG, Eskesen DC, Jairath A, et al. Lactobacillus fermentum ( $\mathrm{PCC}(\mathrm{R})$ ) supplementation and gastrointestinal and respiratory-tract illness symptoms: a randomised control trial in athletes. Nutr J. (2011) 10:30. doi: 10.1186/1475-2891-10-30

43. Peake JM, Neubauer O, Della Gatta PA, Nosaka K. Muscle damage and inflammation during recovery from exercise. J Appl Physiol. (2017) 122:55970. doi: 10.1152/japplphysiol.00971.2016

44. Henning PC, Park BS, Kim JS. Physiological decrements during sustained military operational stress. Mil Med. (2011) 176:991-7. doi: 10.7205/MILMED-D-11-00053

45. Ticinesi A, Lauretani F, Milani C, Nouvenne A, Tana C, Del Rio D, et al. Aging gut microbiota at the cross-road between nutrition, physical frailty, and sarcopenia: is there a gut-muscle axis? Nutrients. (2017) 9:E1303. doi: 10.3390/nu9121303

46. Grosicki GJ, Fielding RA, Lustgarten MS. Gut microbiota contribute to age-related changes in skeletal muscle size, composition, and function: biological basis for a gut-muscle axis. Calcif Tissue Int. (2018) 102:43342. doi: 10.1007/s00223-017-0345-5

47. Jager R, Purpura M, Farmer S, Cash HA, Keller D. Probiotic Bacillus coagulans GBI-30, 6086 improves protein absorption and utilization. Probiotics Antimicrob Proteins. (2018) 10:6115. doi: 10.1007/s12602-017-9354-y

48. Gepner Y, Hoffman JR, Shemesh E, Stout JR, Church DD, Varanoske AN, et al. Combined effect of Bacillus coagulans GBI-30, 6086 and $\mathrm{HMB}$ supplementation on muscle integrity and cytokine response during intense military training. J Appl Physiol. (2017) 123:11-8. doi: 10.1152/japplphysiol.01116.2016 
49. Jager R, Shields KA, Lowery RP, De Souza EO, Partl JM, Hollmer C, et al. Probiotic Bacillus coagulans GBI-30, 6086 reduces exerciseinduced muscle damage and increases recovery. PeerJ. (2016) 4:e2276. doi: 10.7717/peerj.2276

50. Jager R, Purpura M, Stone JD, Turner SM, Anzalone AJ, Eimerbrink MJ, et al. Probiotic Streptococcus thermophilus FP4 and Bifidobacterium breve BR03 supplementation attenuates performance and range-ofmotion decrements following muscle damaging exercise. Nutrients. (2016) 8:E642. doi: 10.3390/nu8100642

51. Molfino A, Gioia G, Rossi Fanelli F, Muscaritoli M. Beta-hydroxybeta-methylbutyrate supplementation in health and disease: a systematic review of randomized trials. Amino Acids. (2013) 45:1273-92. doi: 10.1007/s00726-013-1592-z

52. King S, Glanville J, Sanders ME, Fitzgerald A, Varley D. Effectiveness of probiotics on the duration of illness in healthy children and adults who develop common acute respiratory infectious conditions: a systematic review and meta-analysis. Br J Nutr. (2014) 112:4154. doi: $10.1017 /$ S0007114514000075

53. Nieman DC. Immune response to heavy exertion. J Appl Physiol. (1997) 82:1385-94. doi: 10.1152/jappl.1997.82.5.1385

54. Nieman DC, Henson DA, Austin MD, Sha W. Upper respiratory tract infection is reduced in physically fit and active adults. Br J Sports Med. (2011) 45:987-92. doi: 10.1136/bjsm.2010.077875

55. Gleeson M, Bishop NC. URI in athletes: are mucosal immunity and cytokine responses key risk factors? Exerc Sport Sci Rev. (2013) 41:14853. doi: 10.1097/JES.0b013e3182956ead

56. Sanchez JL, Cooper MJ, Myers CA, Cummings JF, Vest KG, Russell KL, et al. Respiratory infections in the U.S. military: recent experience and control. Clin Microbiol Rev. (2015) 28:743-800. doi: 10.1128/CMR.00039-14

57. Clancy RL, Gleeson M, Cox A, Callister R, Dorrington M, D'este C, et al. Reversal in fatigued athletes of a defect in interferon gamma secretion after administration of Lactobacillus acidophilus. Br J Sports Med. (2006) 40:351-4. doi: 10.1136/bjsm.2005.024364

58. Moreira A, Kekkonen R, Korpela R, Delgado L, Haahtela T. Allergy in marathon runners and effect of Lactobacillus GG supplementation on allergic inflammatory markers. Respir Med. (2007) 101:1123-31. doi: 10.1016/j.rmed.2006.11.015

59. Tiollier E, Chennaoui M, Gomez-Merino D, Drogou C, Filaire E, Guezennec $\mathrm{CY}$. Effect of a probiotics supplementation on respiratory infections and immune and hormonal parameters during intense military training. Mil Med. (2007) 172:1006-11. doi: 10.7205/MILMED.172.9.1006

60. Kekkonen RA, Vasankari TJ, Vuorimaa T, Haahtela T, Julkunen I, Korpela $\mathrm{R}$. The effect of probiotics on respiratory infections and gastrointestinal symptoms during training in marathon runners. Int J Sport Nutr Exerc Metab. (2007) 17:352-63. doi: 10.1123/ijsnem.17.4.352

61. Martarelli D, Verdenelli MC, Scuri S, Cocchioni M, Silvi S, Cecchini C, et al. Effect of a probiotic intake on oxidant and antioxidant parameters in plasma of athletes during intense exercise training. Curr Microbiol. (2011) 62:1689-96. doi: 10.1007/s00284-011-9915-3

62. Gleeson M, Bishop NC, Oliveira M, Tauler P. Daily probiotic's (Lactobacillus casei Shirota) reduction of infection incidence in athletes. Int J Sport Nutr Exerc Metab. (2011) 21:55-64. doi: 10.1123/ijsnem.21.1.55

63. Gleeson M, Bishop NC, Oliveira M, Mccauley T, Tauler P, Lawrence C. Effects of a Lactobacillus salivarius probiotic intervention on infection, cold symptom duration and severity, and mucosal immunity in endurance athletes. Int J Sport Nutr Exerc Metab. (2012) 22:235-42. doi: 10.1123/ijsnem.22.4.235

64. Gill SK, Allerton DM, Ansley-Robson P, Hemmings K, Cox M, Costa RJ. Does short-term high dose probiotic supplementation containing Lactobacillus casei attenuate exertional-heat stress induced endotoxaemia and cytokinaemia? Int J Sport Nutr Exerc Metab. (2016) 26:26875. doi: 10.1123/ijsnem.2015-0186

65. Valimaki IA, Vuorimaa T, Ahotupa M, Kekkonen R, Korpela R, Vasankari T. Decreased training volume and increased carbohydrate intake increases oxidized LDL levels. Int J Sports Med. (2012) 33:2916. doi: $10.1055 / \mathrm{s}-0031-1291223$

66. West NP, Horn PL, Pyne DB, Gebski VJ, Lahtinen SJ, Fricker PA, et al. Probiotic supplementation for respiratory and gastrointestinal illness symptoms in healthy physically active individuals. Clin Nutr. (2014) 33:5817. doi: 10.1016/j.clnu.2013.10.002

67. Haywood BA, Black KE, Baker D, Mcgarvey J, Healey P, Brown RC. Probiotic supplementation reduces the duration and incidence of infections but not severity in elite rugby union players. J Sci Med Sport. (2014) 17:35660. doi: 10.1016/j.jsams.2013.08.004

68. O'brien KV, Stewart LK, Forney LA, Aryana KJ, Prinyawiwatkul W, Boeneke CA. The effects of postexercise consumption of a kefir beverage on performance and recovery during intensive endurance training. J Dairy Sci. (2015) 98:7446-9. doi: 10.3168/jds.2015-9392

69. Roberts JD, Suckling CA, Peedle GY, Murphy JA, Dawkins TG, Roberts MG. An exploratory investigation of endotoxin levels in novice long distance triathletes, and the effects of a multi-strain probiotic/prebiotic, antioxidant intervention. Nutrients. (2016) 8:E733. doi: 10.3390/nu8110733

70. Gleeson M, Bishop NC, Struszczak L. Effects of Lactobacillus casei Shirota ingestion on common cold infection and herpes virus antibodies in endurance athletes: a placebo-controlled, randomized trial. Eur J Appl Physiol. (2016) 116:1555-63. doi: 10.1007/s00421-016-3415-x

71. Michalickova D, Minic R, Dikic N, Andjelkovic M, Kostic-Vucicevic M, Stojmenovic T, et al. Lactobacillus helveticus Lafti L10 supplementation reduces respiratory infection duration in a cohort of elite athletes: a randomized, double-blind, placebo-controlled trial. Appl Physiol Nutr Metab. (2016) 41:782-9. doi: 10.1139/apnm-2015-0541

72. Krumbeck JA, Rasmussen HE, Hutkins RW, Clarke J, Shawron $\mathrm{K}$, Keshavarzian A, et al. Probiotic Bifidobacterium strains and galactooligosaccharides improve intestinal barrier function in obese adults but show no synergism when used together as synbiotics. Microbiome. (2018) 6:121. doi: 10.1186/s40168-018-0494-4

73. Strasser B, Geiger D, Schauer M, Gostner JM, Gatterer H, Burtscher $\mathrm{M}$, et al. Probiotic supplements beneficially affect tryptophan-kynurenine metabolism and reduce the incidence of upper respiratory tract infections in trained athletes: a randomized, double-blinded, placebo-controlled trial. Nutrients. (2016) 8:E752. doi: 10.3390/nu8110752

74. Michalickova D, Kotur-Stevuljevic J, Miljkovic M, Dikic N, Kostic-Vucicevic M, Andjelkovic M, et al. Effects of probiotic supplementation on selected parameters of blood prooxidant-antioxidant balance in elite athletes: a double-blind randomized placebo-controlled study. J Hum Kinet. (2018) 64:111-22. doi: 10.1515/hukin-2017-0203

75. Komano Y, Shimada K, Naito H, Fukao K, Ishihara Y, Fujii T, et al. Efficacy of heat-killed Lactococcus lactis JCM 5805 on immunity and fatigue during consecutive high intensity exercise in male athletes: a randomized, placebo-controlled, double-blinded trial. J Int Soc Sports Nutr. (2018) 15:39. doi: 10.1186/s12970-018-0244-9

76. Lambert GP. Intestinal barrier dysfunction, endotoxemia, and gastrointestinal symptoms: the 'canary in the coal mine' during exercise-heat stress? Med Sport Sci. (2008) 53:61-73. doi: 10.1159/000151550

77. Lambert GP. Stress-induced gastrointestinal barrier dysfunction and its inflammatory effects. J Anim Sci. (2009) 87:E1018. doi: $10.2527 /$ jas.2008-1339

78. Bischoff SC, Barbara G, Buurman W, Ockhuizen T, Schulzke JD, Serino $\mathrm{M}$, et al. Intestinal permeability-a new target for disease prevention and therapy. BMC Gastroenterol. (2014) 14:189. doi: 10.1186/s12876-014-0 189-7

79. Karl JP, Hatch AM, Arcidiacono SM, Pearce SC, Pantoja-Feliciano IG, Doherty LA, et al. Effects of psychological, environmental and physical stressors on the gut microbiota. Front Microbiol. (2018) 9:2013. doi: 10.3389/fmicb.2018.02013

80. Li X, Kan EM, Lu J, Cao Y, Wong RK, Keshavarzian A, et al. Combat-training increases intestinal permeability, immune activation and gastrointestinal symptoms in soldiers. Aliment Pharmacol Ther. (2013) 37:799-809. doi: 10.1111/apt.12269

81. Li X, Wilder-Smith CH, Kan ME, Lu J, Cao Y, Wong RK. Combat-training stress in soldiers increases S100B, a marker of increased blood-brainbarrier permeability, and induces immune activation. Neuro Endocrinol Lett. (2014) 35:58-63.

82. Karl JP, Margolis LM, Madslien EH, Murphy NE, Castellani JW, Gundersen $\mathrm{Y}$, et al. Changes in intestinal microbiota composition and metabolism coincide with increased intestinal permeability in young adults under 
prolonged physiological stress. Am J Physiol Gastrointest Liver Physiol. (2017) 312:G559-71. doi: 10.1152/ajpgi.00066.2017

83. Walker LA, Zambraski EJ, Williams RF. Widespread use of prescription nonsteroidal anti-inflammatory drugs among U.S. Army active duty soldiers. Mil Med. (2017) 182:e1709-12. doi: 10.7205/MILMED-D-16-00183

84. Endo H, Higurashi T, Hosono K, Sakai E, Sekino Y, Iida H, et al. Efficacy of Lactobacillus casei treatment on small bowel injury in chronic low-dose aspirin users: a pilot randomized controlled study. J Gastroenterol. (2011) 46:894-905. doi: 10.1007/s00535-011-0410-1

85. Lamprecht M, Bogner S, Schippinger G, Steinbauer K, Fankhauser F, Hallstroem S, et al. Probiotic supplementation affects markers of intestinal barrier, oxidation, and inflammation in trained men; a randomized, double-blinded, placebo-controlled trial. J Int Soc Sports Nutr. (2012) 9:45. doi: 10.1186/1550-2783-9-45

86. Gotteland M, Cruchet S, Verbeke S. Effect of Lactobacillus ingestion on the gastrointestinal mucosal barrier alterations induced by indometacin in humans. Aliment Pharmacol Ther. (2001) 15:11-7. doi: 10.1046/j.1365-2036.2001.00898.x

87. Floch MH, Walker WA, Sanders ME, Nieuwdorp M, Kim AS, Brenner DA, et al. Recommendations for probiotic Use-2015 update: proceedings and consensus opinion. J Clin Gastroenterol. (2015) 49(Suppl. 1):S6973. doi: 10.1097/MCG.0000000000000420

88. Forbes A, Escher J, Hebuterne X, Klek S, Krznaric Z, Schneider S, et al. ESPEN guideline: clinical nutrition in inflammatory bowel disease. Clin Nutr. (2017) 36:321-47. doi: 10.1016/j.clnu.2016.12.027

89. Cryan JF, Dinan TG. Mind-altering microorganisms: the impact of the gut microbiota on brain and behaviour. Nat Rev Neurosci. (2012) 13:70112. doi: $10.1038 / \mathrm{nrn} 3346$

90. Dinan TG, Cryan JF. Regulation of the stress response by the gut microbiota: implications for psychoneuroendocrinology. Psychoneuroendocrinology. (2012) 37:1369-78. doi: 10.1016/j.psyneuen.2012.03.007

91. Rao AV, Bested AC, Beaulne TM, Katzman MA, Iorio C, Berardi JM, et al. A randomized, double-blind, placebo-controlled pilot study of a probiotic in emotional symptoms of chronic fatigue syndrome. Gut Pathog. (2009) 1:6. doi: 10.1186/1757-4749-1-6

92. Akkasheh G, Kashani-Poor Z, Tajabadi-Ebrahimi M, Jafari P, Akbari $\mathrm{H}$, Taghizadeh $\mathrm{M}$, et al. Clinical and metabolic response to probiotic administration in patients with major depressive disorder: a randomized, double-blind, placebo-controlled trial. Nutrition. (2016) 32:315-20. doi: 10.1016/j.nut.2015.09.003

93. Chang YK, Labban JD, Gapin JI, Etnier JL. The effects of acute exercise on cognitive performance: a meta-analysis. Brain Res. (2012) 1453:87101. doi: $10.1016 /$ j.brainres.2012.02.068

94. Sandi C. Stress and cognition. Wiley Interdiscipl Rev Cogn Sci. (2013) 4:24561. doi: $10.1002 /$ wcs. 1222

95. Pontifex MB, Mcgowan AL, Chandler MC, Gwizdala KL, Parks AC, Fenn K, et al. A primer on investigating the after effects of acute bouts of physical activity on cognition. Psychol Sport Exerc. (2019) 40:122. doi: 10.1016/j.psychsport.2018.08.015

96. Lichtenberger EO, Kaufman AS. Essentials of WAIS-IV Assessment. Hoboken, NJ: John Wiley \& Sons (2009).

97. Kelly JR, Allen AP, Temko A, Hutch W, Kennedy PJ, Farid N, et al. Lost in translation? The potential psychobiotic Lactobacillus rhamnosus (JB-1) fails to modulate stress or cognitive performance in healthy male subjects. Brain Behav Immun. (2017) 61:50-9. doi: 10.1016/j.bbi.2016.1 1.018

98. Lew LC, Hor YY, Yusoff, NA, Choi SB, Yusoff MSB, Liong MT. Probiotic Lactobacillus plantarum P8 alleviated stress and anxiety while enhancing memory and cognition in stressed adults: a randomised, double-blind, placebo-controlled study. Clin Nutr. (2018) 38:2053-64. doi: 10.1016/j.clnu.2018.09.010

99. Allen AP, Hutch W, Borre YE, Kennedy PJ, Temko A, Boylan G, et al. Bifidobacterium longum 1714 as a translational psychobiotic: modulation of stress, electrophysiology and neurocognition in healthy volunteers. Transl Psychiatry. (2016) 6:e939. doi: 10.1038/tp.2016.191

100. Benton D, Williams C, Brown A. Impact of consuming a milk drink containing a probiotic on mood and cognition. Eur J Clin Nutr. (2007) 61:355-61. doi: 10.1038/sj.ejcn.1602546
101. Chong HX, Yusoff, NA., Hor YY, Lew LC, Jaafar MH, Liong MT. Lactobacillus plantarum DR7 alleviates stress and anxiety in adults: a randomised, double-blind, placebo-controlled study. Benef Microbes. (2019) 10:355-73. doi: 10.3920/BM2018.0135

102. Chung Y-C, Jin H-M, Cui Y, Kim DS, Jung JM, Park J-I, et al. Fermented milk of Lactobacillus helveticus IDCC3801 improves cognitive functioning during cognitive fatigue tests in healthy older adults. J Funct Foods. (2014) 10:465-74. doi: 10.1016/j.jff.2014.07.007

103. Papalini S, Michels F, Kohn N, Wegman J, Hemert SV, Roelofs K, et al. Stress matters: a double-blind, randomized controlled trial on the effects of a multispecies probiotic on neurocognition. Neurobiol Stress. (2018) 10:263673.

104. Nyberg L, Lovden M, Riklund K, Lindenberger U, Backman L. Memory aging and brain maintenance. Trends Cogn Sci. (2012) 16:292-305. doi: 10.1016/j.tics.2012.04.005

105. Miyake A, Friedman NP, Emerson MJ, Witzki AH, Howerter A, Wager TD. The unity and diversity of executive functions and their contributions to complex "Frontal Lobe" tasks: a latent variable analysis. Cogn Psychol. (2000) 41:49-100. doi: 10.1006/cogp.1999.0734

106. Schoofs D, Preuss D, Wolf OT. Psychosocial stress induces working memory impairments in an n-back paradigm. Psychoneuroendocrinology. (2008) 33:643-53. doi: 10.1016/j.psyneuen.2008.02.004

107. Schoofs D, Wolf OT, Smeets T. Cold pressor stress impairs performance on working memory tasks requiring executive functions in healthy young men. Behav Neurosci. (2009) 123:1066-75. doi: 10.1037/a0016980

108. Scherer KR. Psychological models of emotion. In Borod JC editor. The Neuropsychology of Emotion. New York, NY: Oxford University Press (2000). p. 137-62.

109. Scherer KR. What are emotions? And how can they be measured? Soc. Sci. Inform. (2005) 44:695-729. doi: 10.1177/0539018405058216

110. Marotta A, Sarno E, Del Casale A, Pane M, Mogna L, Amoruso A, et al. Effects of probiotics on cognitive reactivity, mood, sleep quality. Front Psychiatry. (2019) 10:164. doi: 10.3389/fpsyt.2019.00164

111. Messaoudi M, Lalonde R, Violle N, Javelot H, Desor D, Nejdi A, et al. Assessment of psychotropic-like properties of a probiotic formulation (Lactobacillus helveticus R0052 and Bifidobacterium longum R0175) in rats and human subjects. Br J Nutr. (2011) 105:755-64. doi: 10.1017/S0007114510004319

112. Messaoudi M, Violle N, Bisson JF, Desor D, Javelot H, Rougeot C. Beneficial psychological effects of a probiotic formulation (Lactobacillus helveticus R0052 and Bifidobacterium longum R0175) in healthy human volunteers. Gut Microbes. (2011) 2:256-61. doi: 10.4161/gmic.2.4.16108

113. Noorwali EA, Beaumont JD, Corfe BM, Owen L. The effects of probiotic supplementation on emotional memory and pain response. Proc Nutr Soc. (2017) 76:E6. doi: 10.1017/S0029665117000064

114. Owen L, Reinders M, Narramore R, Marsh AMR, Gar Lui F, Baron R, et al. A double blind, placebo controlled, randomised pilot trial examining the effects of probiotic administration on mood and cognitive function. Proc Nutr Soc. (2014) 73:E29. doi: 10.1017/S0029665114000433

115. Steenbergen L, Sellaro R, Van Hemert S, Bosch JA, Colzato LS. A randomized controlled trial to test the effect of multispecies probiotics on cognitive reactivity to sad mood. Brain Behav Immun. (2015) 48:25864. doi: 10.1016/j.bbi.2015.04.003

116. Wang H, Braun C, Murphy EF, Enck P. Bifidobacterium longum $1714^{\mathrm{TM}}$ strain modulates brain activity of healthy volunteers during social stress. $\mathrm{Am}$ J Gastroenterol. (2019) 114:1152-62. doi: 10.14309/ajg.0000000000000203

117. Papalini S, Michels F, Kohn N, Wegman J, Van Hemert S, Roelofs $\mathrm{K}$, et al. Stress matters: randomized controlled trial on the effect of probiotics on neurocognition. Neurobiol Stress. (2019) 10:100141. doi: 10.1016/j.ynstr.2018.100141

118. Guo S, Dipietro LA. Factors affecting wound healing. J Dent Res. (2010) 89:219-29. doi: 10.1177/0022034509359125

119. Rodrigues M, Kosaric N, Bonham CA, Gurtner GC. Wound healing: a cellular perspective. Physiol Rev. (2019) 99:665706. doi: 10.1152/physrev.00067.2017

120. Gouin JP, Kiecolt-Glaser JK. The impact of psychological stress on wound healing: methods and mechanisms. Immunol Allergy Clin North Am. (2011) 31:81-93. doi: 10.1016/j.iac.2010.09.010 
121. Murray CK. Field wound care: prophylactic antibiotics. Wilderness Environ Med. (2017) 28:S90-102. doi: 10.1016/j.wem.2016.12.009

122. Allison-Aipa TS, Ritter C, Sikes P, Ball S. The impact of deployment on the psychological health status, level of alcohol consumption, and use of psychological health resources of postdeployed U.S. Army Reserve soldiers. Mil Med. (2010) 175:630-7. doi: 10.7205/MILMED-D-09-00212

123. Lukic J, Chen V, Strahinic I, Begovic J, Lev-Tov H, Davis SC, et al. Probiotics or pro-healers: the role of beneficial bacteria in tissue repair. Wound Repair Regen. (2017) 25:912-22. doi: 10.1111/wrr.12607

124. Tsiouris CG, Tsiouri MG. Human microflora, probiotics and wound healing. Wound Med. (2017) 19:33-8. doi: 10.1016/j.wndm.2017.09.006

125. Yang $\mathrm{Z}, \mathrm{Wu} \mathrm{Q}$, Liu Y, Fan D. Effect of perioperative probiotics and synbiotics on postoperative infections after gastrointestinal surgery: a systematic review with meta-analysis. J Parenter Enteral Nutr. (2017) 41:1051-62. doi: 10.1177/0148607116629670

126. Kasatpibal N, Whitney JD, Saokaew S, Kengkla K, Heitkemper MM, Apisarnthanarak A. Effectiveness of probiotic, prebiotic, and synbiotic therapies in reducing postoperative complications: a systematic review and network meta-analysis. Clin Infect Dis. (2017) 64:S153-60. doi: 10.1093/cid/cix114

127. Kotzampassi K, Stavrou G, Damoraki G, Georgitsi M, Basdanis G, Tsaousi $\mathrm{G}$, et al. A four-probiotics regimen reduces postoperative complications after colorectal surgery: a randomized, double-blind, placebo-controlled study. World J Surg. (2015) 39:2776-83. doi: 10.1007/s00268-015-3071-z

128. Blanchet-Rethore S, Bourdes V, Mercenier A, Haddar CH, Verhoeven PO, Andres P. Effect of a lotion containing the heat-treated probiotic strain Lactobacillus johnsonii NCC 533 on Staphylococcus aureus colonization in atopic dermatitis. Clin Cosmet Investig Dermatol. (2017) 10:24957. doi: 10.2147/CCID.S135529

129. Gueniche A, Knaudt B, Schuck E, Volz T, Bastien P, Martin R, et al. Effects of nonpathogenic gram-negative bacterium Vitreoscilla filiformis lysate on atopic dermatitis: a prospective, randomized, doubleblind, placebo-controlled clinical study. Br J Dermatol. (2008) 159:135763. doi: 10.1111/j.1365-2133.2008.08836.x

130. Gueniche A, Philippe D, Bastien P, Reuteler G, Blum S, Castiel-Higounenc I, et al. Randomised double-blind placebo-controlled study of the effect of Lactobacillus paracasei NCC 2461 on skin reactivity. Benef Microbes. (2014) 5:137-45. doi: 10.3920/BM2013.0001

131. Lee DE, Huh CS, Ra J, Choi ID, Jeong JW, Kim SH, et al. Clinical evidence of effects of Lactobacillus plantarum HY7714 on skin aging: a randomized, double blind, placebo-controlled study. J Microbiol Biotechnol. (2015) 25:2160-8. doi: 10.4014/jmb.1509.09021

132. Mohseni S, Bayani M, Bahmani F, Tajabadi-Ebrahimi M, Bayani MA, Jafari $\mathrm{P}$, et al. The beneficial effects of probiotic administration on wound healing and metabolic status in patients with diabetic foot ulcer: a randomized, double-blind, placebo-controlled trial. Diabetes Metab Res Rev. (2018) 34:e2970. doi: 10.1002/dmrr.2970

133. Ogawa M, Saiki A, Matsui Y, Tsuchimoto N, Nakakita Y, Takata Y, et al. Effects of oral intake of heat-killed Lactobacillus brevis SBC8803 (SBL88) on dry skin conditions: a randomized, double-blind, placebo-controlled study. Exp Ther Med. (2016) 12:3863-72. doi: 10.3892/etm.2016.3862

134. Peral MC, Martinez MA, Valdez JC. Bacteriotherapy with Lactobacillus plantarum in burns. Int Wound J. (2009) 6:7381. doi: 10.1111/j.1742-481X.2008.00577.x

135. Peral MC, Rachid MM, Gobbato NM, Huaman Martinez MA, Valdez JC. Interleukin-8 production by polymorphonuclear leukocytes from patients with chronic infected leg ulcers treated with Lactobacillus plantarum. Clin Microbiol Infect. (2010) 16:281-6. doi: 10.1111/j.1469-0691.2009.02793.x

136. Smith TJ, Wilson MA, Young AJ, Montain SJ. A suction blister model reliably assesses skin barrier restoration and immune response. J Immunol Methods. (2015) 417:124-30. doi: 10.1016/j.jim.2015.01.002

137. Smith TJ, Wilson MA, Karl JP, Orr J, Smith CD, Cooper AD, et al. Impact of sleep restriction on local immune response and skin barrier restoration with and without "multinutrient" nutrition intervention. J Appl Physiol. (2018) 124:190-200. doi: 10.1152/japplphysiol.00547.2017

138. Tsiouris CG, Kelesi M, Vasilopoulos G, Kalemikerakis I, Papageorgiou EG. The efficacy of probiotics as pharmacological treatment of cutaneous wounds: meta-analysis of animal studies. Eur J Pharm Sci. (2017) 104:2309. doi: 10.1016/j.ejps.2017.04.002
139. Center DAVBI. DoD Worldwide Numbers for TBI [Online]. (2019). Available online at: https://dvbic.dcoe.mil/files/tbi-numbers/worldwide-totals2000-2018Q1-total_jun-21-2018_v1.0_2018-07-26_0.pdf (accessed May 2, 2019).

140. Houlden A, Goldrick M, Brough D, Vizi ES, Lenart N, Martinecz B, et al. Brain injury induces specific changes in the caecal microbiota of mice via altered autonomic activity and mucoprotein production. Brain Behav Immun. (2016) 57:10-20. doi: 10.1016/j.bbi.2016.04.003

141. Gautam A, Kumar R, Chakraborty N, Muhie S, Hoke A, Hammamieh R, et al. Altered fecal microbiota composition in all male aggressor-exposed rodent model simulating features of post-traumatic stress disorder. J Neurosci Res. (2018) 96:1311-23. doi: 10.1002/jnr.24229

142. Nicholson SE, Watts LT, Burmeister DM, Merrill D, Scroggins S, Zou Y, et al. Moderate traumatic brain injury alters the gastrointestinal microbiome in a time-dependent manner. Shock. (2019) 52:240-8. doi: 10.1097/SHK.0000000000001211

143. Brenner LA, Stearns-Yoder KA, Hoffberg AS, Penzenik ME, Starosta $\mathrm{AJ}$, Hernandez TD, et al. Growing literature but limited evidence: a systematic review regarding prebiotic and probiotic interventions for those with traumatic brain injury and/or posttraumatic stress disorder. Brain Behav Immun. (2017) 65:57-67. doi: 10.1016/j.bbi.2017. 06.003

144. Brenner LA, Hoisington AJ, Stearns-Yoder KA, Stamper CE, Heinze JD, Postolache TT, et al. Military-related exposures, social determinants of health, and dysbiosis: The United States-Veteran Microbiome Project (US-VMP). Front Cell Infect Microbiol. (2018) 8:400. doi: $10.3389 /$ fcimb. 2018.00400

145. Zhu CS, Grandhi R, Patterson TT, Nicholson SE. A review of traumatic brain injury and the gut microbiome: insights into novel mechanisms of secondary brain injury and promising targets for neuroprotection. Brain Sci. (2018) 8:113. doi: $10.3390 /$ brainsci8060113

146. Falcao De Arruda IS, De Aguilar-Nascimento. J.E. Benefits of early enteral nutrition with glutamine and probiotics in brain injury patients. Clin Sci. (2004) 106:287-92. doi: 10.1042/CS20030251

147. Tan M, Zhu JC, Du J, Zhang LM, Yin HH. Effects of probiotics on serum levels of Th1/Th2 cytokine and clinical outcomes in severe traumatic braininjured patients: a prospective randomized pilot study. Crit Care. (2011) 15:R290. doi: 10.1186/cc10579

148. Manzanares W, Lemieux M, Langlois PL, Wischmeyer PE. Probiotic and synbiotic therapy in critical illness: a systematic review and meta-analysis. Crit Care. (2016) 19:262. doi: 10.1186/s13054-016-1434-y

149. Collado MC, Vinderola G, Salminen S. Postbiotics: facts and open questions. A position paper on the need for a consensus definition. Benef Microbes. (2019) 10:711-9. doi: 10.3920/BM2019.0015

150. Doron S, Snydman DR. Risk and safety of probiotics. Clin Infect Dis. (2015) 60(Suppl. 2):S129-34. doi: 10.1093/cid/civ085

Disclaimers: JK, GG, KR, and JS: All opinions or assertions contained herein are the private views of the authors and are not to be construed as official or reflecting the views of the U.S. Army or Department of Defense. Any citations of commercial organizations and trade names in this report do not constitute an official Department of the Army endorsement or approval of the products or services of these organizations. Approved for public release; distribution is unlimited. RA and KM: The views expressed in this article reflect the results of research conducted by the author and do not necessarily reflect the official policy or position of the Department of the Navy, Department of Defense, nor the United States Government.

Conflict of Interest: The authors declare that the research was conducted in the absence of any commercial or financial relationships that could be construed as a potential conflict of interest.

Copyright $\odot 2020$ Agans, Giles, Goodson, Karl, Leyh, Mumy, Racicot and Soares. This is an open-access article distributed under the terms of the Creative Commons Attribution License (CC BY). The use, distribution or reproduction in other forums is permitted, provided the original author(s) and the copyright owner $(s)$ are credited and that the original publication in this journal is cited, in accordance with accepted academic practice. No use, distribution or reproduction is permitted which does not comply with these terms. 